\title{
Supporting Information \\ Standard Validation Protocol for Selected Ion Flow Tube Mass Spectrometry \\ Methods Applied to Direct Headspace Analysis of Aqueous Volatile Organic \\ Compounds
}

Mark J. Perkins ${ }^{1}$, Vaughan S. Langford ${ }^{*}, 2$

${ }^{1}$ Anatune Limited, Wellbrook Court, Girton Road, Cambridge, CB3 ONA, United Kingdom

${ }^{2}$ Syft Technologies Limited, 3 Craft Place, Christchurch 8024, New Zealand

* Contributing author: vaughan.langford@syft.com

\section{Table of Contents}

\section{Supplementary Figures}

Figure S1. Optimization of incubation time at an incubation temperature of $60^{\circ} \mathrm{C}$ for selected compounds.

Figure S2. Optimization of the volume of aqueous solution used for the headspace measurements for selected compounds.

Figure S3. Optimization of headspace partitioning using 0 and $10 \% \mathrm{NaCl}$.

Figure S4. Effective speciation of chloroform and DCM using the $\mathrm{OH}^{-}$reagent ion.

Figure S5. Linearity of automated HS-SIFT-MS measurements of standards in the $10-2000 \mathrm{ppb}$ range (in solution) for selected compounds.

\section{Supplementary Tables}

Table S1. Suppliers and purities of chemicals (used without further purification).

Table S2. Reagent ions and primary product ions mass-to charge ratios (in Daltons) used to quantify target compounds.

Table S3. Optimization of incubation temperature from 40 to $80^{\circ} \mathrm{C}$ for selected compounds (by quantitation ion) using automated SIFT-MS.

Table S4. Repeatability (measured using \%RSD) of triplicate aliquots of aqueous solution used for the volume optimization study.

Table S5. Linearity data for each reagent-product ion pair in the method.

Table S6. Data obtained at various low-ppb concentrations (1, 2, 5, $10 \mathrm{ppb})$ in solution for determination of the method LOQ.

Table S7. System precision data for the method.

Table S8. Analytical precision data for the method.

Table S9. Accuracy data (in ppm in solution) for each of the triplicate measurements at 500, 1000, and $1500 \mathrm{ppb}$ concentration in water.

Table S10. Recovery data (\%) for each of the triplicate measurements at 500, 1000, and $1500 \mathrm{ppb}$ concentration in water.

Table S11. Accuracy data (in ppm in solution) obtained in the intermediate precision study (different analyst).

Table S12. Recovery data (\%) obtained in the intermediate precision study (different analyst).

Table S13. Robustness data as assessed using linearity $\left(R^{2}\right)$ and repeatability (\%RSD) measures for two changes to sample flow rate and inlet temperature reduction. 


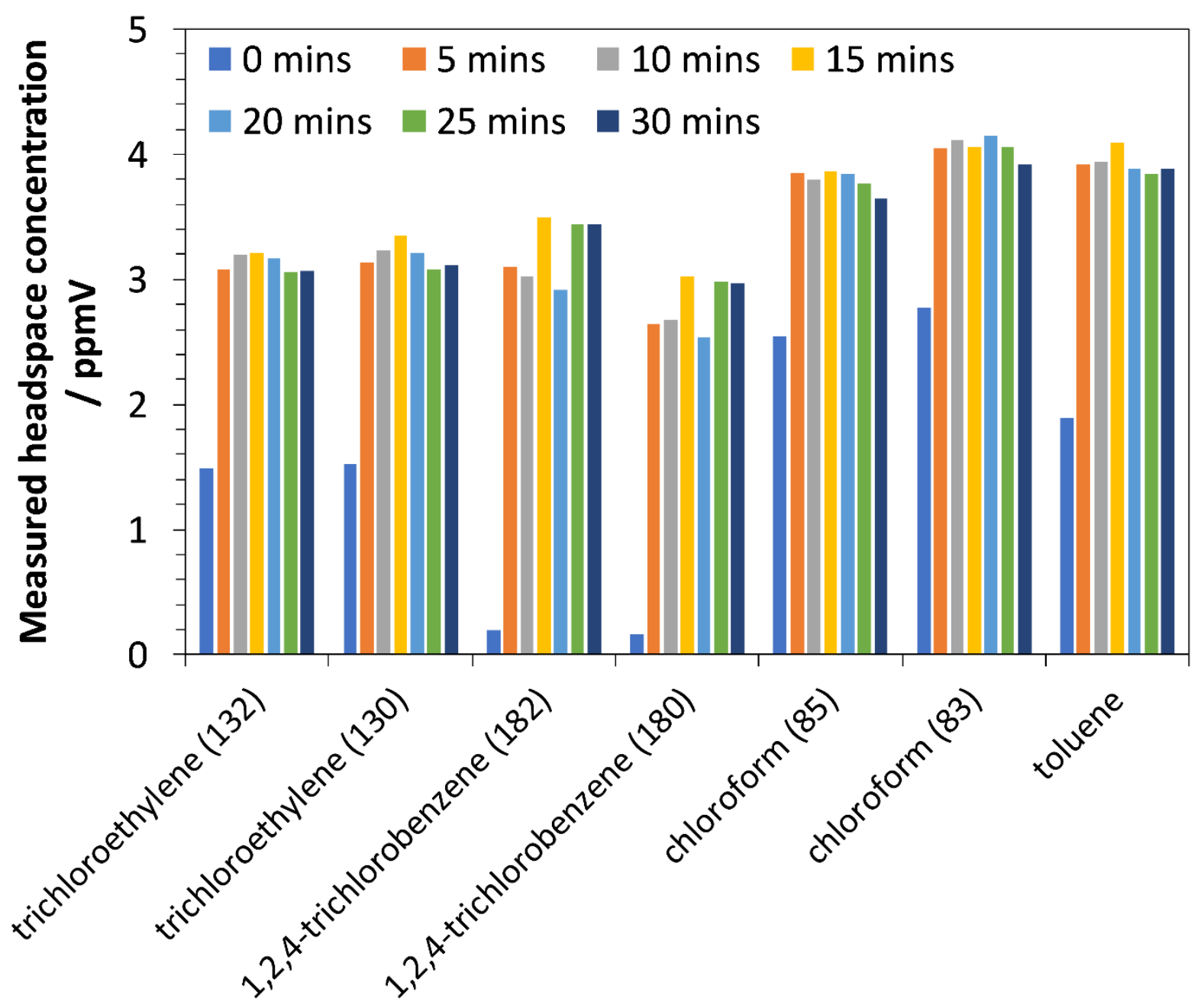

Figure S1. Optimization of incubation time at an incubation temperature of $60^{\circ} \mathrm{C}$ for selected compounds (by quantitation ion) using automated SIFT-MS. 
a)

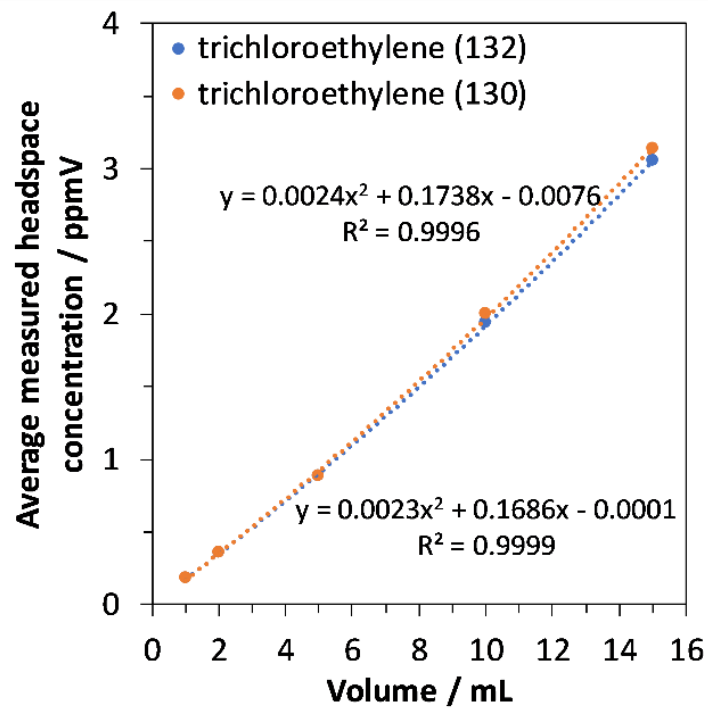

c)

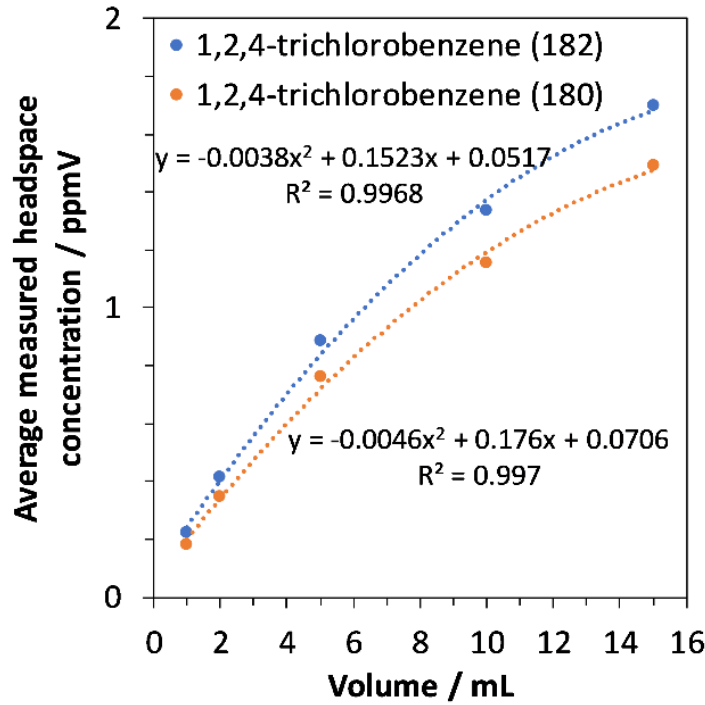

b)

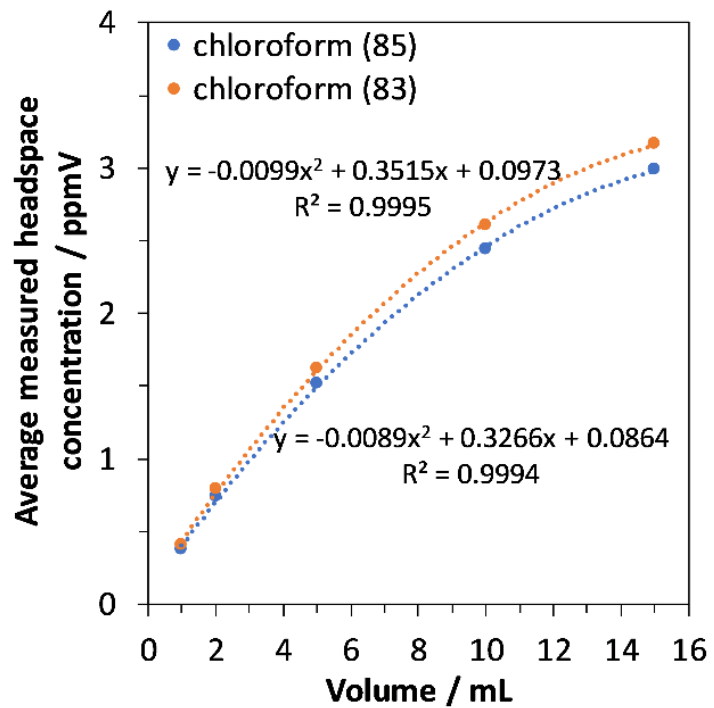

d)

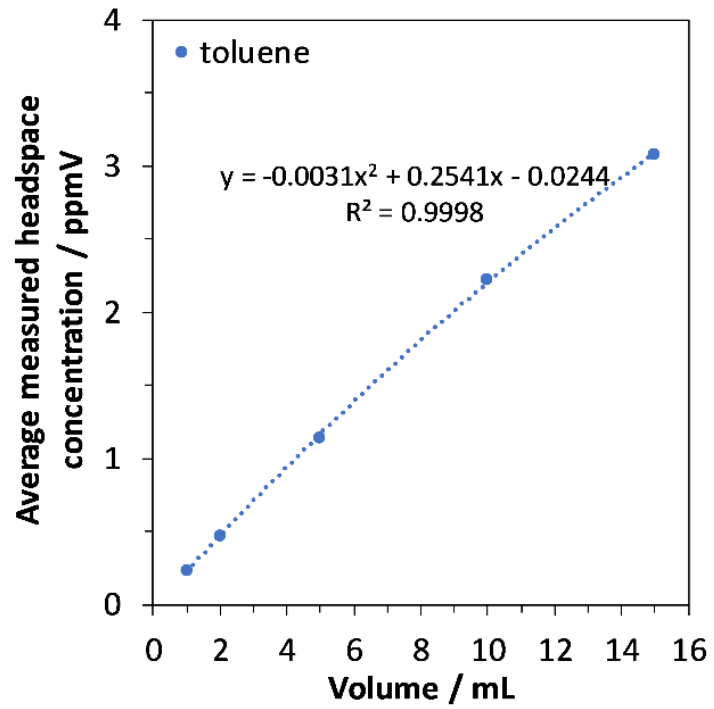

Figure S2. Optimization of the volume of aqueous solution used for the headspace measurements for selected compounds (by quantitation ion). 


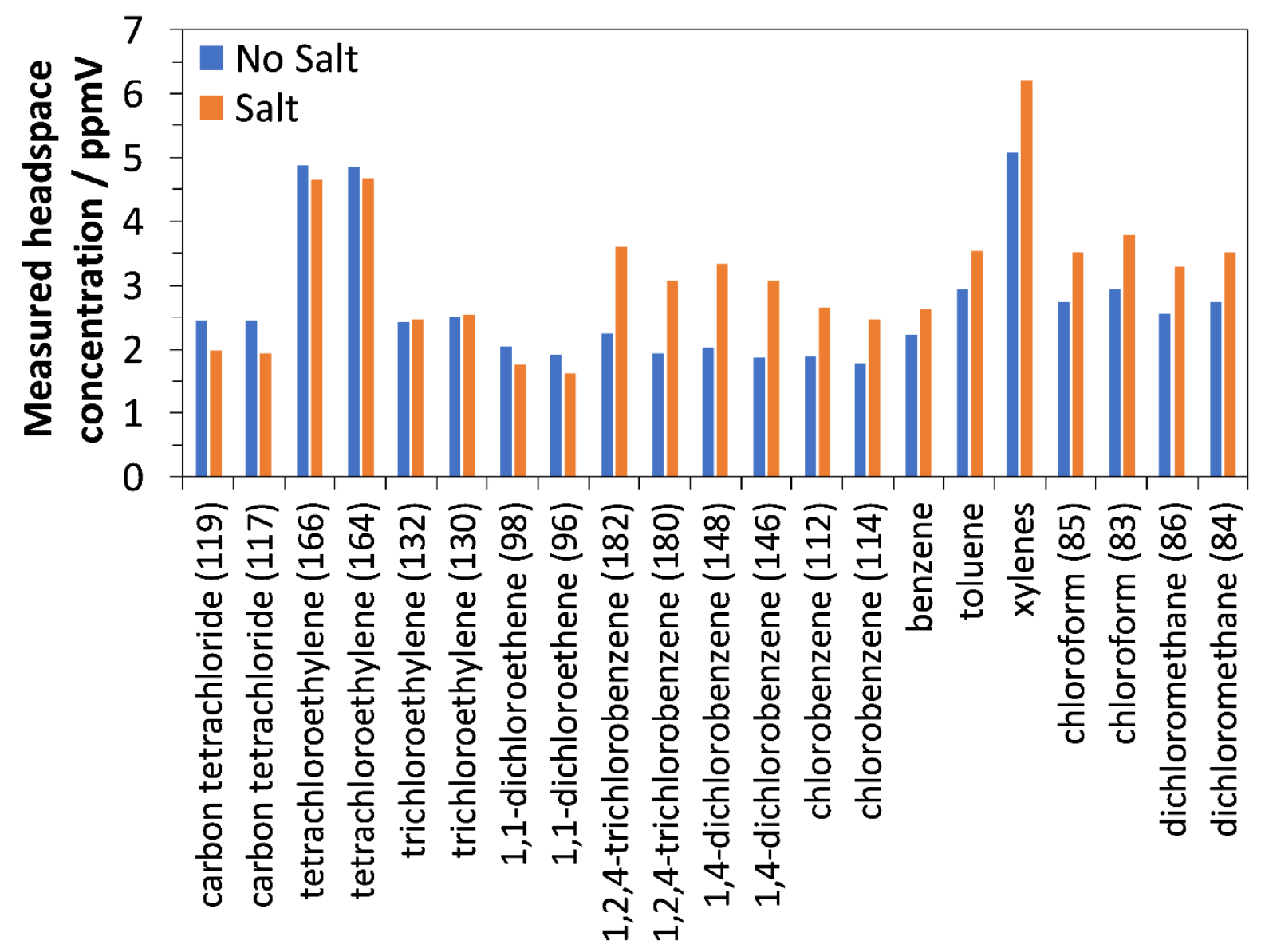

Figure S3. Optimization of headspace partitioning using 0 and $10 \% \mathrm{NaCl}$ for selected compounds. 


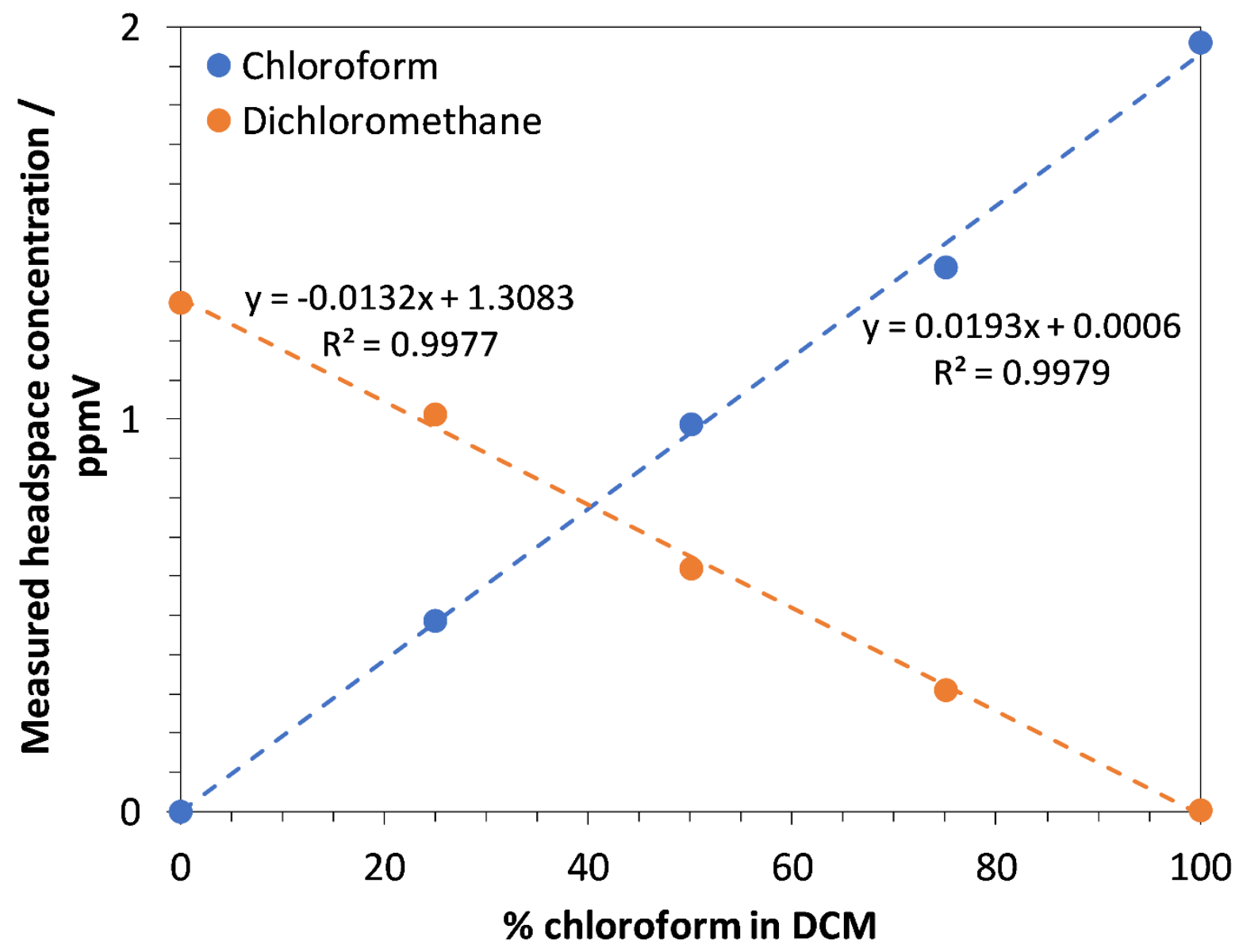

Figure S4. Effective speciation of chloroform and DCM using the $\mathrm{OH}^{-}$reagent ion. 

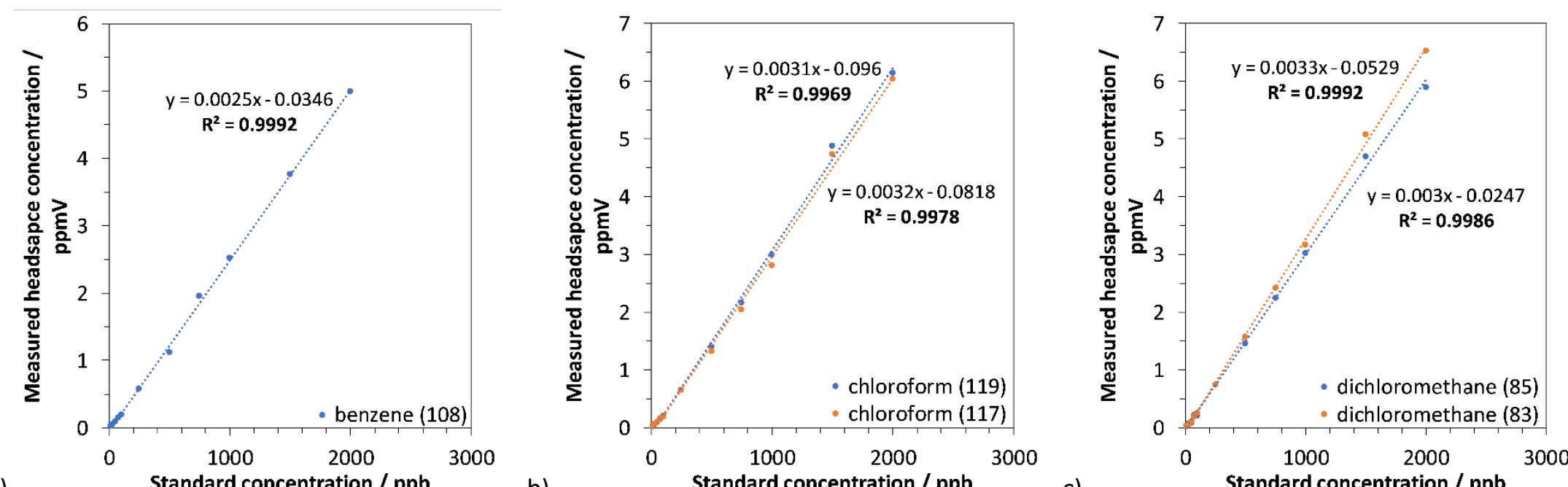

a)

b)

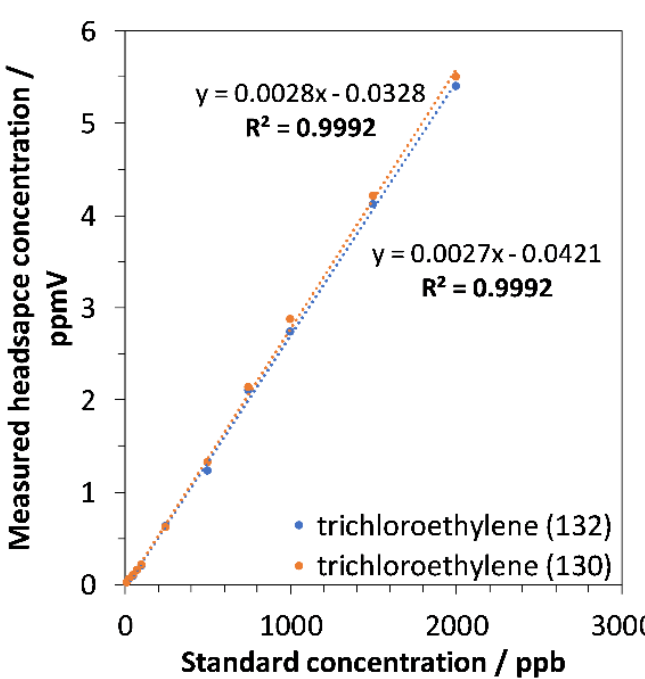
Standard concentration / ppb

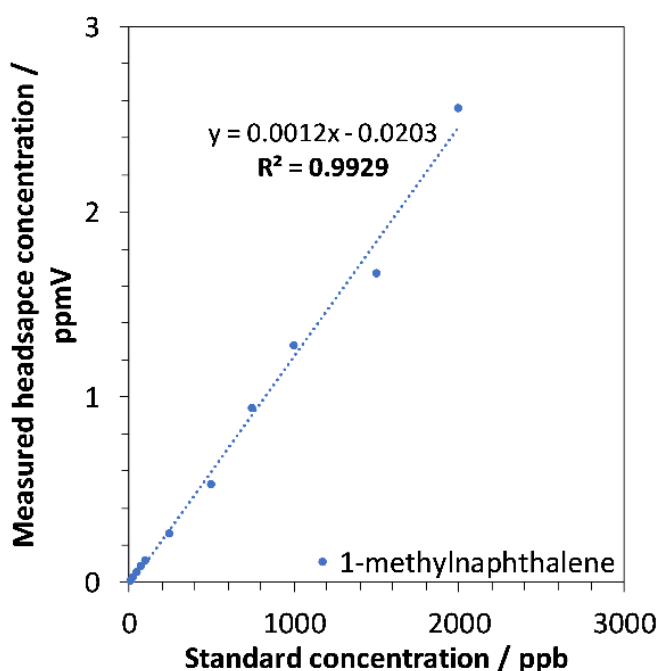

e)

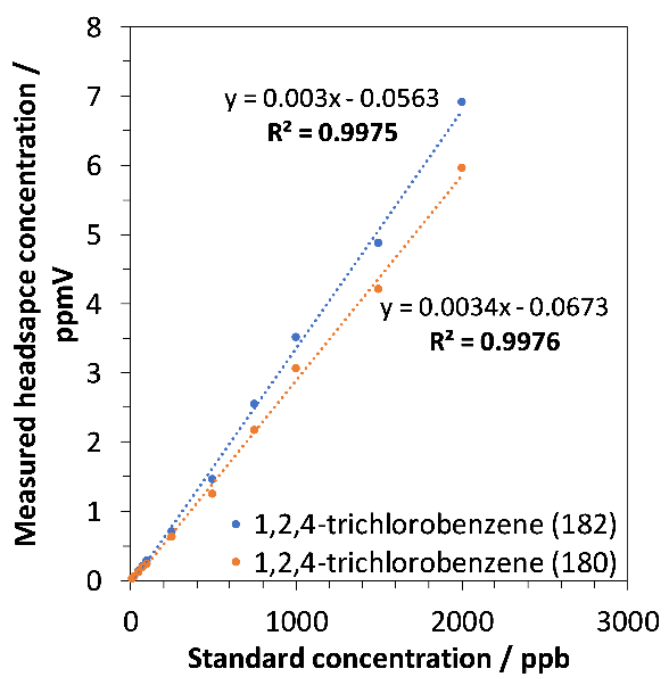

Figure S5. Linearity of automated HS-SIFT-MS measurements of standards in the $10-2000$ ppb range (in solution) for selected compounds: (a) benzene, (b) chloroform, (c) DCM, (d) 1-methylnaphthalene, (e) trichloroethylene, and (f) 1,2,4-trichlorobenzene. Headspace concentrations are in ppmV. 
Supplementary Tables

Table S1. Suppliers and purities of chemicals (used without further purification).

\begin{tabular}{llll} 
Name & CAS No. & Supplier & Purity or Grade \\
\hline Benzene & $71-43-2$ & Fluka & $>99.9 \%$ \\
Carbon tetrachloride & $56-23-5$ & Sigma Aldrich & $99 \%$ \\
Chlorobenzene & $108-90-7$ & Sigma Aldrich & $99.8 \%$ \\
Chloroform & $67-66-3$ & Sigma Aldrich & $>99 \%$ \\
1,4-Dichlorobenzene & $106-46-7$ & Sigma Aldrich & $99 \%$ \\
1,1-Dichloroethene & $75-35-4$ & Sigma Aldrich & $99 \%$ \\
Dichloromethane (DCM) & $75-09-2$ & Sigma Aldrich & $>99.8 \%$ \\
Dimethyl sulfoxide & $67-68-5$ & Sigma Aldrich & $\geq 99.5 \%$ \\
Ethylbenzene & $100-41-4$ & Sigma Aldrich & $99.8 \%$ \\
Isooctane & $540-84-1$ & Sigma Aldrich & $>99.5 \%$ \\
1-Methylnaphthalene & $90-12-0$ & Sigma Aldrich & $97 \%$ \\
Naphthalene & $91-20-3$ & Sigma Aldrich & $99 \%$ \\
Tetrachloroethylene & $127-18-4$ & Sigma Aldrich & $>99 \%$ \\
Toluene & $108-88-3$ & Aldrich & $99 \%$ \\
1,2,4-Trichlorobenzene & $120-82-1$ & Sigma Aldrich & $>99 \%$ \\
Trichloroethylene & $79-01-6$ & Sigma Aldrich & $>99 \%$ \\
1,3,5-Trimethylbenzene & $108-67-8$ & Sigma Aldrich & $99 \%$ \\
Xylene (all isomers; $m$ - used) & $108-38-3$ & Sigma Aldrich & $>99 \%$ \\
\hline & & &
\end{tabular}


Table S2. Reagent ions and primary product ions mass-to charge ratios (in Daltons) used to quantify target compounds, with branching ratio in parenthesis. For simplicity, other unused ion products of each compound are shown. More details are given in the article.

\begin{tabular}{|c|c|c|c|c|}
\hline \multirow[t]{2}{*}{ Compound } & \multicolumn{3}{|c|}{ Reagent and product ions used } & \multirow{2}{*}{$\begin{array}{l}\text { Reference for reaction ion } \\
\text { chemistry (see footnote) }\end{array}$} \\
\hline & $\mathrm{OH}^{-}$ & $\mathrm{NO}^{+}$ & $\mathrm{O}_{2}^{+}$ & \\
\hline \multirow[t]{2}{*}{ Benzene } & & $78(76 \%)$ & & 1 \\
\hline & & $108(24 \%)$ & & \\
\hline \multirow[t]{2}{*}{ Carbon tetrachloride } & & & $117(44 \%)$ & 1 \\
\hline & & & $119(42 \%)$ & \\
\hline \multirow[t]{2}{*}{ Chlorobenzene } & & & $112(75 \%)$ & 3 \\
\hline & & & $114(25 \%)$ & \\
\hline \multirow[t]{2}{*}{ Chloroform } & $117(44 \%)$ & & & 1,4 \\
\hline & $119(42 \%)$ & & & \\
\hline \multirow[t]{2}{*}{ 1,4-Dichlorobenzene } & & & $146(60 \%)$ & 5 \\
\hline & & & $148(35 \%)$ & \\
\hline \multirow[t]{2}{*}{ 1,1-Dichloroethylene } & & & $96(60 \%)$ & 6 \\
\hline & & & $98(35 \%)$ & \\
\hline \multirow[t]{2}{*}{ Dichloromethane } & $83(56 \%)$ & & & 1,4 \\
\hline & $85(38 \%)$ & & & \\
\hline \multirow[t]{2}{*}{ Ethylbenzene } & & $106(100 \%)$ & $91(30 \%)$ & 1 \\
\hline & & & $106(70 \%)$ & \\
\hline Isooctane & & $113(55 \%)$ & & 7 \\
\hline 1-Methylnaphthalene & & $142(100 \%)$ & & 8 \\
\hline Naphthalene & & $128(100 \%)$ & & 8 \\
\hline \multirow[t]{2}{*}{ Tetrachloroethylene } & & & $164(32 \%)$ & 2 \\
\hline & & & $166(42 \%)$ & \\
\hline Toluene & & $92(100 \%)$ & & 1 \\
\hline \multirow[t]{2}{*}{ 1,2,4-Trichlorobenzene } & & & $180(47 \%)$ & 7 \\
\hline & & & $182(44 \%)$ & \\
\hline \multirow[t]{2}{*}{ Trichloroethylene } & & & $130(44 \%)$ & 2 \\
\hline & & & $132(42 \%)$ & \\
\hline 1,3,5-Trimethylbenzene & & $120(100 \%)$ & & 1 \\
\hline \multirow[t]{2}{*}{ Xylene (total of all isomers) } & & $106(100 \%)$ & $91(75 \%)$ & 1 \\
\hline & & & $106(25 \%)$ & \\
\hline
\end{tabular}

* References: (1) Spanel, P.; Smith, D. SIFT studies of the reactions of $\mathrm{H}_{3} \mathrm{O}^{+}, \mathrm{NO}^{+}$and $\mathrm{O}_{2}{ }^{+}$with several aromatic and aliphatic hydrocarbons. Int. J Mass Spectrom. 1998, 181, 1-10. (2) Spanel, P.; Smith, D. Selected ion flow tube studies of the reactions of $\mathrm{H}_{3} \mathrm{O}^{+}, \mathrm{NO}^{+}$, and $\mathrm{O}_{2}{ }^{+}$with some chloroalkanes and chloroalkenes. Int. J. Mass Spectrom. 1999, 184, 175-181. (3) Spanel, P.; Smith, D. Selected ion flow tube studies of the reactions of $\mathrm{H}_{3} \mathrm{O}^{+}$, $\mathrm{NO}^{+}$, and $\mathrm{O}_{2}{ }^{+}$with several aromatic and aliphatic monosubstituted halocarbons. Int. J. Mass Spectrom. 1999, 189, 213-223. (4) Perkins, M. Speciation of Dichloromethane and Chloroform Using Negative Ion SIFT-MS, Anatune application note AS233, 2020. Available at www.anatune.co.uk. (5) Syft Technologies Limited. SIFTMS Compound Library, 2011. (6) Syft Technologies Limited. SIFT-MS Compound Library, 2006. Syft Technologies Limited. (7) SIFT-MS Compound Library, 2014. (8) Milligan, D.B.; Wilson, P.F.; Freeman, C.G.; Meot-Ner, M.; McEwan, M.J. J. Phys. Chem. A, 106, 9745-9755, 2002. 
Table S3. Optimization of incubation temperature from 40 to $80^{\circ} \mathrm{C}$ for selected compounds (by quantitation ion) using automated SIFT-MS. The mean and standard deviation (SD) of the headspace concentration measurements are shown in ppmV. The percent relative standard deviation is also shown (\%RSD). The final SIFT-MS method was not utilized in this phase of method development.

\begin{tabular}{|c|c|c|c|c|c|c|c|c|c|c|c|c|c|c|c|c|c|c|c|c|c|c|}
\hline 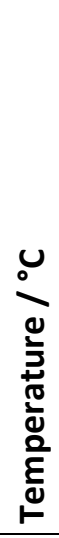 & 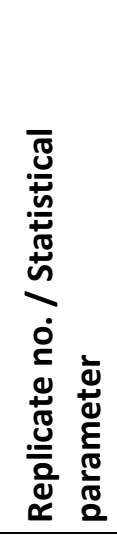 & 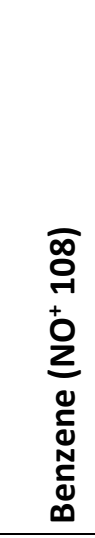 & 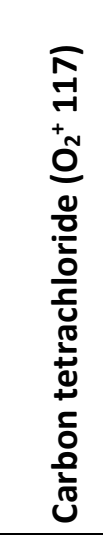 & 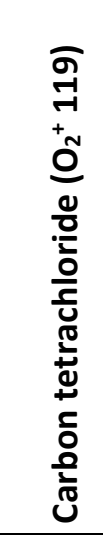 & 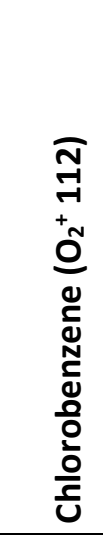 & 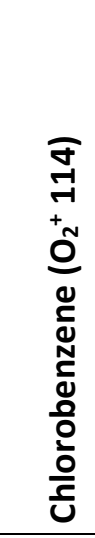 & 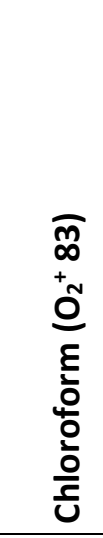 & 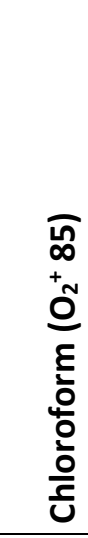 & 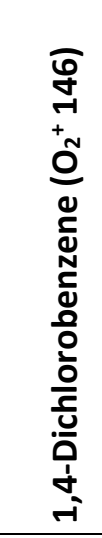 & 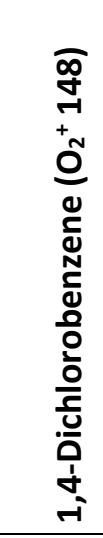 & 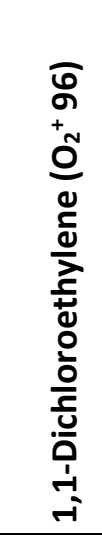 & 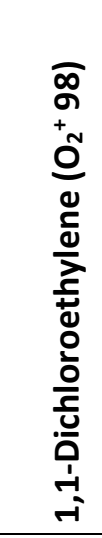 & 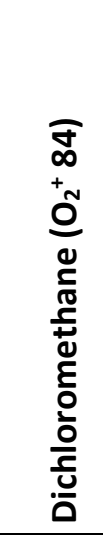 & 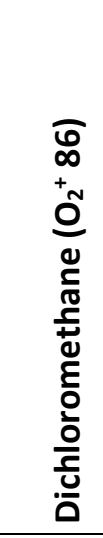 & 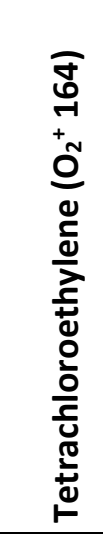 & 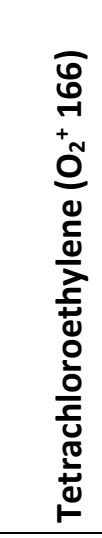 & 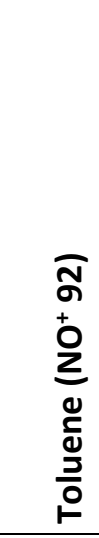 & 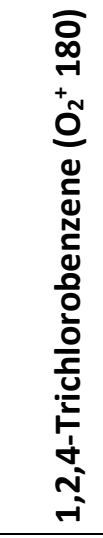 & 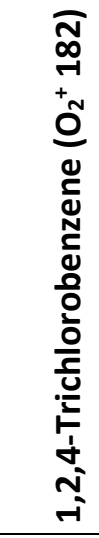 & 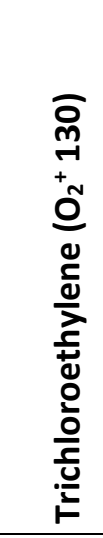 & 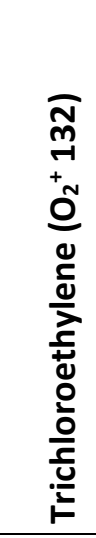 & 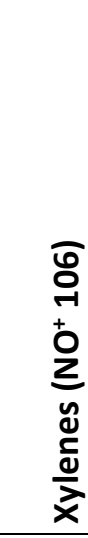 \\
\hline \multirow[t]{6}{*}{40} & 1 & 2.38 & 2.64 & 2.66 & 1.10 & 1.04 & 2.07 & 1.95 & 0.800 & 0.863 & 2.33 & 2.48 & 1.94 & 1.82 & 3.86 & 3.94 & 2.00 & 0.538 & 0.596 & 2.07 & 1.99 & 3.05 \\
\hline & 2 & 2.33 & 2.56 & 2.51 & 1.07 & 1.03 & 2.03 & 1.88 & 0.825 & 0.887 & 2.22 & 2.27 & 1.91 & 1.76 & 3.72 & 3.81 & 1.94 & 0.574 & 0.665 & 2.04 & 1.93 & 3.03 \\
\hline & 3 & 2.14 & 2.73 & 2.73 & 1.13 & 1.07 & 2.06 & 1.96 & 0.836 & 0.877 & 2.32 & 2.50 & 1.93 & 1.83 & 4.12 & 4.22 & 2.07 & 0.570 & 0.648 & 2.13 & 2.07 & 3.28 \\
\hline & Mean & 2.28 & 2.64 & 2.63 & 1.10 & 1.04 & 2.05 & 1.93 & 0.821 & 0.875 & 2.29 & 2.42 & 1.93 & 1.80 & 3.90 & 3.99 & 2.00 & 0.561 & 0.636 & 2.08 & 1.99 & 3.12 \\
\hline & $S D$ & 0.103 & 0.069 & 0.092 & 0.024 & 0.017 & 0.017 & 0.036 & 0.015 & 0.010 & 0.050 & 0.104 & 0.012 & 0.031 & 0.166 & 0.171 & 0.053 & 0.016 & 0.029 & 0.037 & 0.057 & 0.113 \\
\hline & $\% R S D$ & 4.5 & 2.6 & 3.5 & 2.2 & 1.6 & 0.8 & 1.8 & 1.8 & 1.1 & 2.2 & 4.3 & 0.6 & 1.7 & 4.3 & 4.3 & 2.7 & 2.9 & 4.6 & 1.8 & 2.9 & 3.6 \\
\hline \multirow[t]{6}{*}{50} & 1 & 2.62 & 2.98 & 2.93 & 1.41 & 1.34 & 2.72 & 2.53 & 1.14 & 1.23 & 2.60 & 2.72 & 2.55 & 2.37 & 4.60 & 4.71 & 2.57 & 0.91 & 1.07 & 2.55 & 2.46 & 3.91 \\
\hline & 2 & 2.66 & 2.80 & 2.77 & 1.47 & 1.37 & 2.59 & 2.43 & 1.29 & 1.39 & 2.46 & 2.55 & 2.43 & 2.27 & 4.38 & 4.47 & 2.46 & 1.20 & 1.38 & 2.41 & 2.37 & 3.86 \\
\hline & 3 & 2.40 & 2.96 & 2.96 & 1.48 & 1.43 & 2.73 & 2.54 & 1.27 & 1.34 & 2.51 & 2.76 & 2.55 & 2.37 & 4.60 & 4.65 & 2.61 & 1.09 & 1.29 & 2.56 & 2.52 & 4.12 \\
\hline & Mean & 2.56 & 2.91 & 2.89 & 1.45 & 1.38 & 2.68 & 2.50 & 1.23 & 1.32 & 2.52 & 2.68 & 2.51 & 2.34 & 4.52 & 4.61 & 2.55 & 1.07 & 1.25 & 2.51 & 2.45 & 3.96 \\
\hline & $S D$ & 0.114 & 0.081 & 0.083 & 0.031 & 0.037 & 0.064 & 0.050 & 0.066 & 0.067 & 0.058 & 0.091 & 0.057 & 0.047 & 0.104 & 0.102 & 0.063 & 0.122 & 0.130 & 0.068 & 0.062 & 0.113 \\
\hline & $\% R S D$ & 4.5 & 2.8 & 2.9 & 2.1 & 2.7 & 2.4 & 2.0 & 5.4 & 5.0 & 2.3 & 3.4 & 2.3 & 2.0 & 2.3 & 2.2 & 2.5 & 11.5 & 10.5 & 2.7 & 2.5 & 2.8 \\
\hline \multirow[t]{6}{*}{60} & 1 & 2.70 & 3.32 & 3.31 & 1.91 & 1.75 & 3.25 & 3.04 & 1.70 & 1.90 & 2.89 & 3.08 & 3.04 & 2.84 & 5.40 & 5.49 & 3.12 & 1.74 & 2.02 & 3.02 & 2.92 & 4.99 \\
\hline & 2 & 2.70 & 3.17 & 3.10 & 1.99 & 1.85 & 3.31 & 3.08 & 1.97 & 2.16 & 2.75 & 2.94 & 3.09 & 2.87 & 5.17 & 5.16 & 3.19 & 2.05 & 2.43 & 2.98 & 2.86 & 5.04 \\
\hline & 3 & 2.60 & 3.12 & 3.08 & 1.96 & 1.81 & 3.23 & 3.02 & 1.90 & 2.06 & 2.68 & 2.85 & 3.01 & 2.81 & 5.09 & 5.15 & 3.14 & 1.94 & 2.28 & 2.85 & 2.87 & 5.06 \\
\hline & Mean & 2.67 & 3.20 & 3.16 & 1.95 & 1.80 & 3.26 & 3.05 & 1.85 & 2.04 & 2.77 & 2.96 & 3.05 & 2.84 & 5.22 & 5.26 & 3.15 & 1.91 & 2.24 & 2.95 & 2.88 & 5.03 \\
\hline & $S D$ & 0.047 & 0.085 & 0.104 & 0.033 & 0.041 & 0.034 & 0.025 & 0.114 & 0.107 & 0.087 & 0.095 & 0.033 & 0.024 & 0.131 & 0.158 & 0.029 & 0.128 & 0.169 & 0.073 & 0.026 & 0.029 \\
\hline & $\% R S D$ & 1.8 & 2.7 & 3.3 & 1.7 & 2.3 & 1.0 & 0.8 & 6.2 & 5.2 & 3.2 & 3.2 & 1.1 & 0.9 & 2.5 & 3.0 & 0.9 & 6.7 & 7.6 & 2.5 & 0.9 & 0.6 \\
\hline & 1 & 2.95 & 3.47 & .47 & 2.29 & 2.15 & 3.77 & 3.56 & 2.32 & 2.48 & 3.10 & 3.29 & 3.51 & 3.32 & 5.68 & 5.65 & 3.65 & 2.55 & 2.86 & 3.30 & 3.21 & .75 \\
\hline
\end{tabular}




\begin{tabular}{|c|c|c|c|c|c|c|c|c|c|c|c|c|c|c|c|c|c|c|c|c|c|c|}
\hline 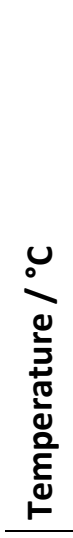 & 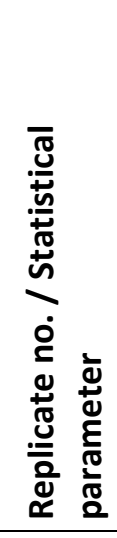 & 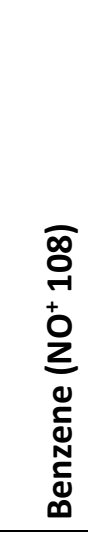 & 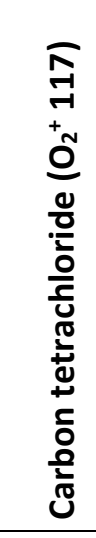 & 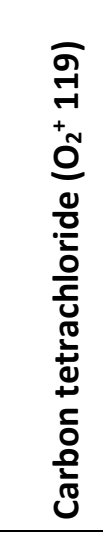 & 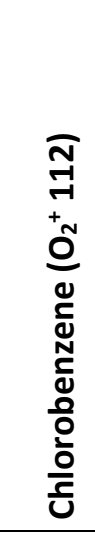 & 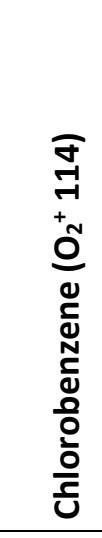 & 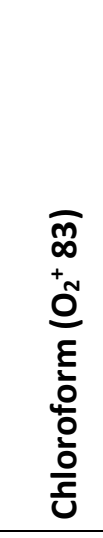 & 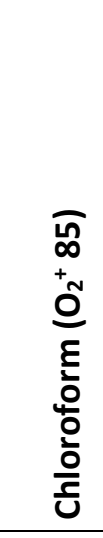 & 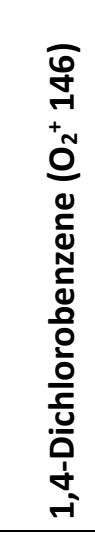 & 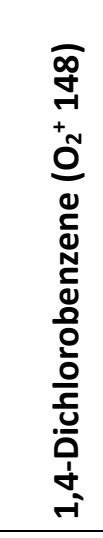 & 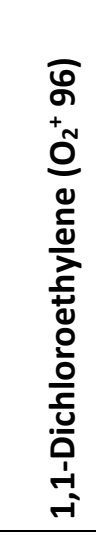 & 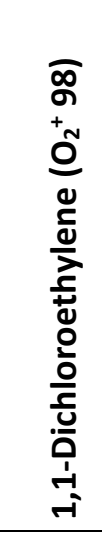 & 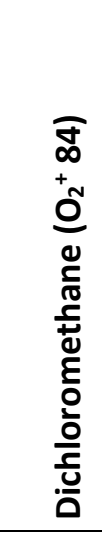 & 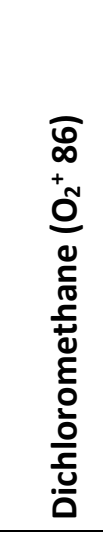 & 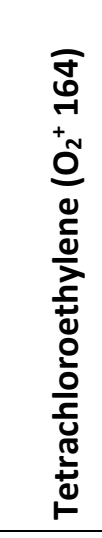 & 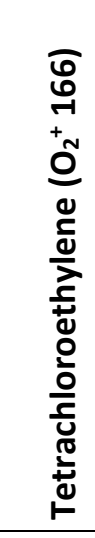 & 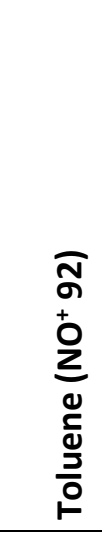 & 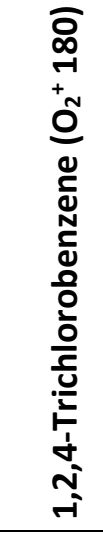 & 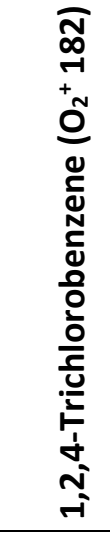 & 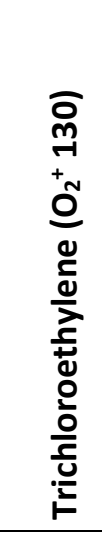 & 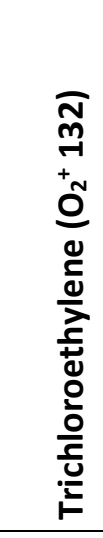 & 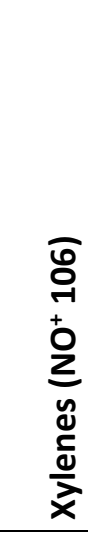 \\
\hline & 2 & 3.14 & 3.61 & 3.59 & 2.52 & 2.36 & 3.89 & 3.74 & 2.65 & 2.94 & 3.15 & 3.34 & 3.63 & 3.48 & 6.13 & 6.20 & 3.89 & 2.97 & 3.43 & 3.55 & 3.40 & 6.35 \\
\hline & 3 & 2.98 & 3.57 & 3.50 & 2.29 & 2.21 & 3.74 & 3.52 & 2.31 & 2.49 & 3.11 & 3.29 & 3.49 & 3.27 & 5.74 & 5.73 & 3.69 & 2.45 & 2.82 & 3.33 & 3.29 & 5.87 \\
\hline & Mean & 3.02 & 3.55 & 3.52 & 2.37 & 2.24 & 3.80 & 3.61 & 2.42 & 2.64 & 3.12 & 3.31 & 3.54 & 3.36 & 5.85 & 5.86 & 3.74 & 2.66 & 3.04 & 3.39 & 3.30 & 5.99 \\
\hline & $S D$ & 0.083 & 0.059 & 0.051 & 0.108 & 0.088 & 0.065 & 0.096 & 0.158 & 0.215 & 0.022 & 0.024 & 0.062 & 0.090 & 0.199 & 0.243 & 0.105 & 0.225 & 0.279 & 0.111 & 0.078 & 0.259 \\
\hline & $\% R S D$ & 2.8 & 1.7 & 1.4 & 4.6 & 3.9 & 1.7 & 2.7 & 6.5 & 8.1 & 0.7 & 0.7 & 1.7 & 2.7 & 3.4 & 4.1 & 2.8 & 8.5 & 9.2 & 3.3 & 2.4 & 4.3 \\
\hline \multirow[t]{6}{*}{80} & 1 & 3.07 & 3.58 & 3.55 & 2.44 & 2.28 & 4.21 & 3.94 & 2.57 & 2.78 & 3.15 & 3.41 & 3.94 & 3.69 & 6.09 & 6.00 & 3.87 & 2.86 & 3.31 & 3.45 & 3.32 & 6.21 \\
\hline & 2 & 3.63 & 3.55 & 3.60 & 3.00 & 2.78 & 5.08 & 4.76 & 3.13 & 3.40 & 3.40 & 3.68 & 4.74 & 4.44 & 5.96 & 5.93 & 4.30 & 3.36 & 3.84 & 3.82 & 3.67 & 6.50 \\
\hline & 3 & 3.23 & 3.40 & 3.42 & 2.57 & 2.46 & 4.45 & 4.14 & 2.68 & 2.99 & 3.09 & 3.33 & 4.15 & 3.86 & 5.89 & 5.84 & 3.90 & 3.01 & 3.51 & 3.50 & 3.34 & 6.19 \\
\hline & Mean & 3.31 & 3.51 & 3.52 & 2.67 & 2.50 & 4.58 & 4.28 & 2.79 & 3.06 & 3.21 & 3.47 & 4.28 & 4.00 & 5.98 & 5.92 & 4.02 & 3.08 & 3.55 & 3.59 & 3.44 & 6.30 \\
\hline & $S D$ & 0.236 & 0.079 & 0.076 & 0.239 & 0.207 & 0.367 & 0.349 & 0.242 & 0.257 & 0.134 & 0.150 & 0.339 & 0.321 & 0.083 & 0.065 & 0.196 & 0.209 & 0.219 & 0.164 & 0.160 & 0.142 \\
\hline & $\% R S D$ & 7.1 & 2.2 & 2.2 & 9.0 & 8.3 & 8.0 & 8.2 & 8.7 & 8.4 & 4.2 & 4.3 & 7.9 & 8.0 & 1.4 & 1.1 & 4.9 & 6.8 & 6.2 & 4.6 & 4.7 & 2.2 \\
\hline
\end{tabular}


Table S4. Repeatability (measured using \%RSD) of triplicate aliquots of aqueous solution used for the volume optimization study. Determined from, headspace measurements (with quantitation reagent/product ion pairs in parentheses). The final SIFT-MS method was not utilized in this phase of method development.

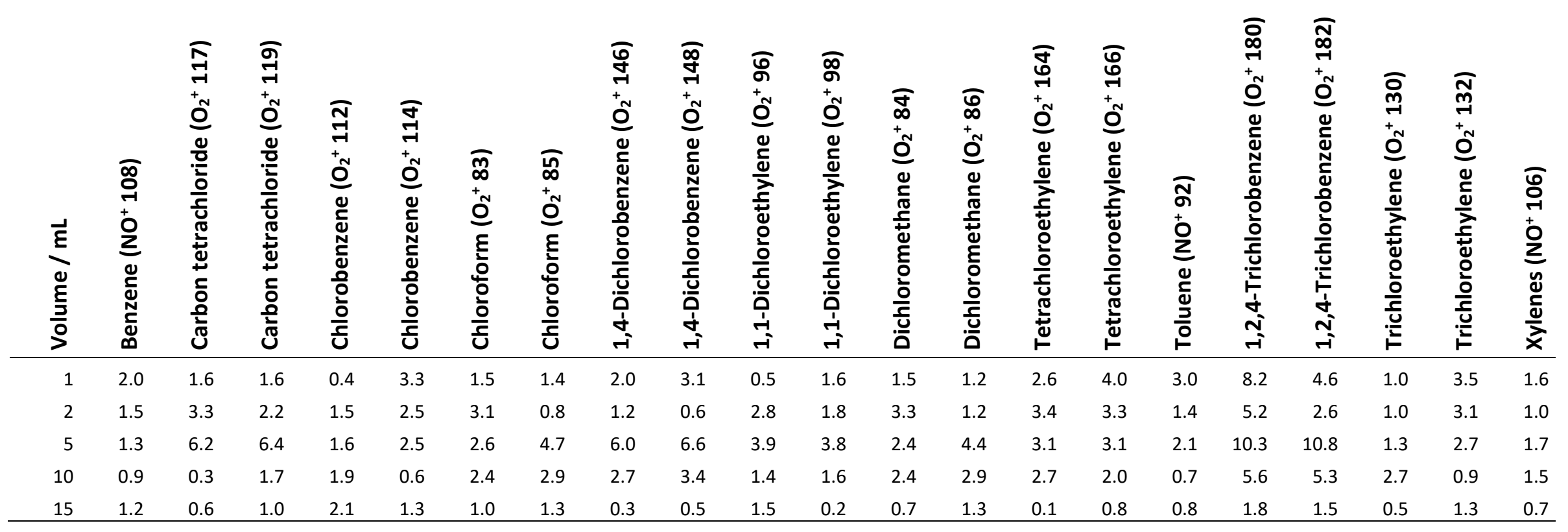


Table S5. Linearity data for each reagent-product ion pair in the method. The regression coefficient $\left(\mathrm{R}^{2}\right)$ shown in the bottom row.

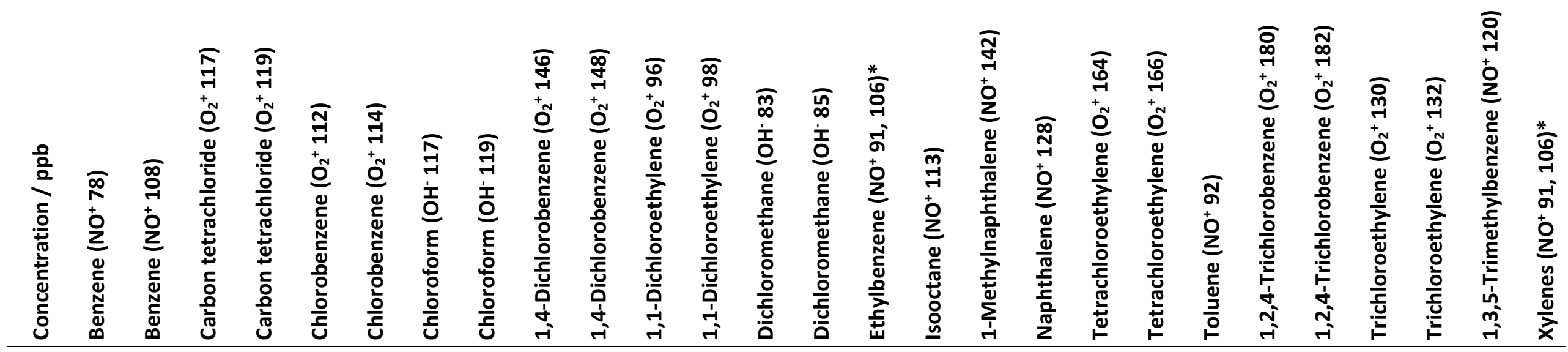

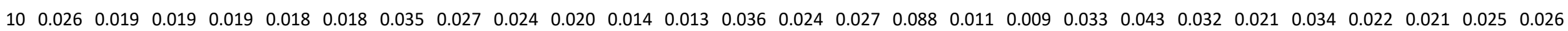

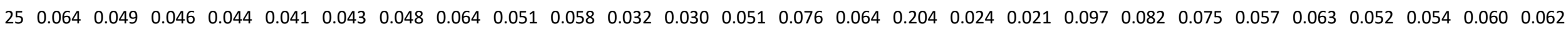

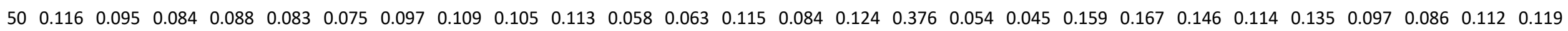
$\begin{array}{llllllllllllllllllllllllllllll}75 & 0.190 & 0.149 & 0.134 & 0.151 & 0.136 & 0.129 & 0.163 & 0.161 & 0.163 & 0.191 & 0.095 & 0.101 & 0.198 & 0.216 & 0.203 & 0.617 & 0.088 & 0.071 & 0.262 & 0.268 & 0.231 & 0.191 & 0.203 & 0.158 & 0.152 & 0.184 & 0.192\end{array}$

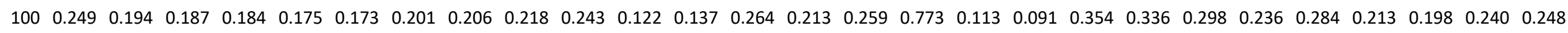

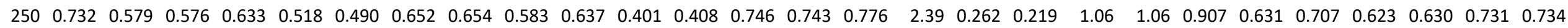

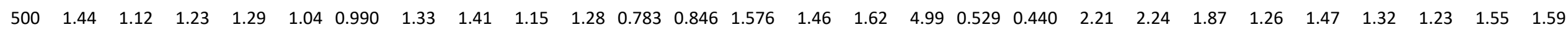
$\begin{array}{lllllllllllllllllllllllllllll}750 & 2.44 & 1.95 & 2.06 & 2.09 & 1.82 & 1.66 & 2.05 & 2.17 & 1.97 & 2.17 & 1.29 & 1.44 & 2.426 & 2.24 & 2.69 & 7.88 & 0.940 & 0.755 & 3.75 & 3.71 & 3.12 & 2.18 & 2.55 & 2.14 & 2.10 & 2.51 & 2.53\end{array}$ $\begin{array}{llllllllllllllllllllllllllllllll}1000 & 3.21 & 2.52 & 2.68 & 2.77 & 2.48 & 2.30 & 2.81 & 3.00 & 2.73 & 2.94 & 1.71 & 1.85 & 3.176 & 3.03 & 3.72 & 9.72 & 1.28 & 1.05 & 4.92 & 4.94 & 4.20 & 3.06 & 3.51 & 2.87 & 2.74 & 3.47 & 3.56\end{array}$ $\begin{array}{lllllllllllllllllllllllllllll}1500 & 4.68 & 3.76 & 4.35 & 4.54 & 3.58 & 3.33 & 4.74 & 4.88 & 3.74 & 4.10 & 2.66 & 2.83 & 5.076 & 4.69 & 5.51 & 16.3 & 1.67 & 1.37 & 7.49 & 7.43 & 6.09 & 4.21 & 4.87 & 4.21 & 4.12 & 5.13 & 5.19\end{array}$ \begin{tabular}{llllllllllllllllllllllllllllll}
2000 & 6.00 & 4.99 & 5.51 & 5.69 & 4.90 & 4.60 & 6.04 & 6.15 & 5.26 & 5.70 & 3.26 & 3.50 & 6.536 & 5.89 & 7.44 & 19.3 & 2.56 & 2.08 & 9.48 & 9.62 & 8.14 & 5.96 & 6.92 & 5.50 & 5.40 & 6.98 & 7.26 \\
\hline
\end{tabular}

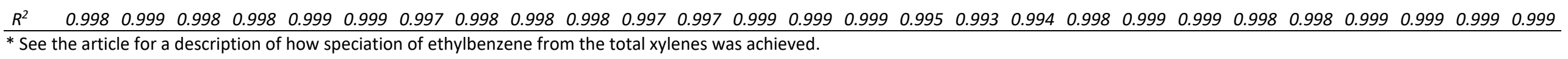


Table S6. Data obtained at various low-ppb concentrations (1, 2, 5, $10 \mathrm{ppb})$ in solution for determination of the method LOQ. Mean, standard deviation (SD), and RSD (in \%) are shown in for the triplicate measurements made at each concentration.

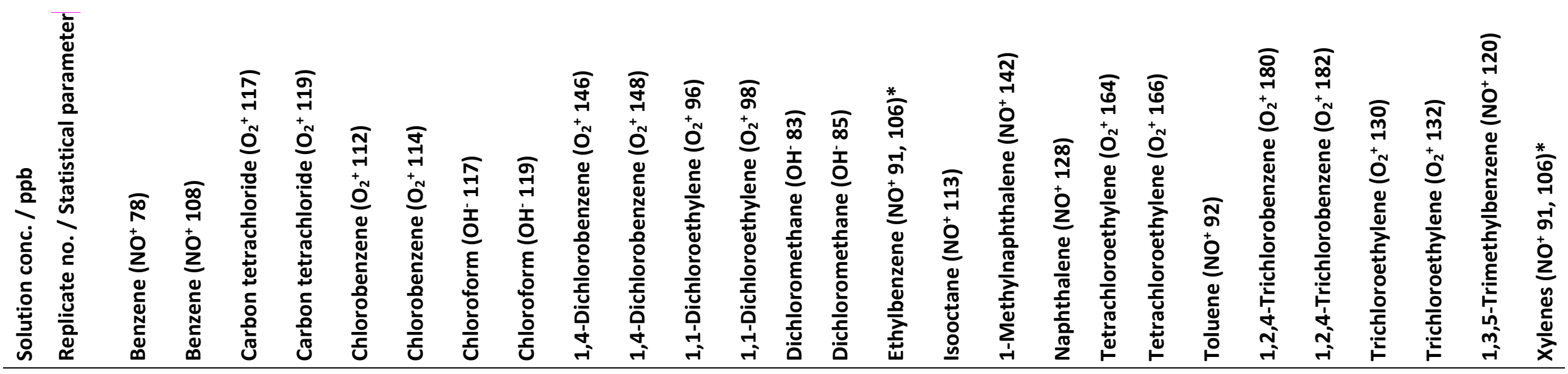

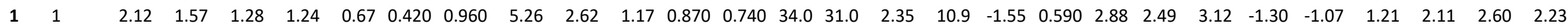

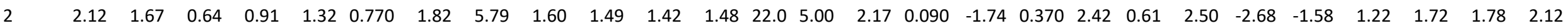

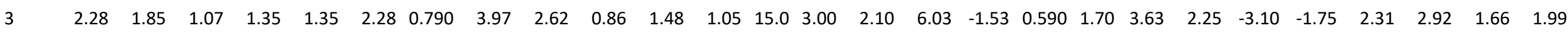

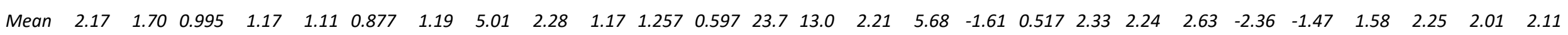

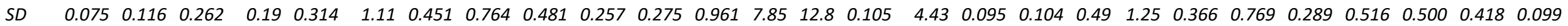

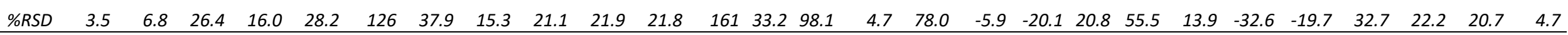

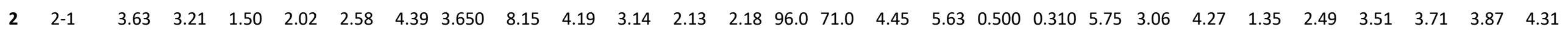

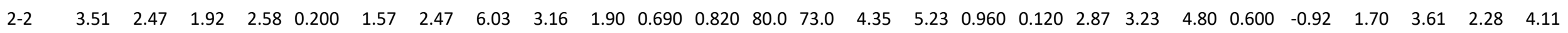

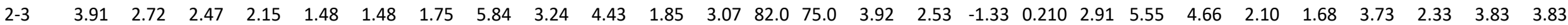

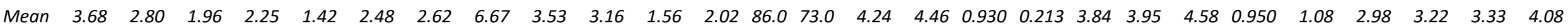

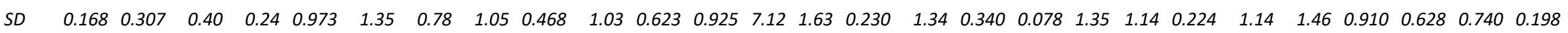

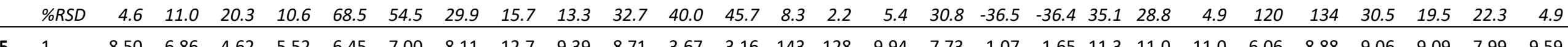

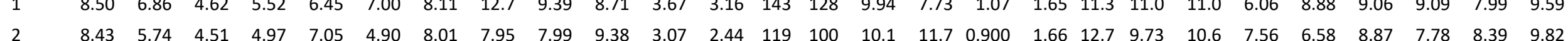

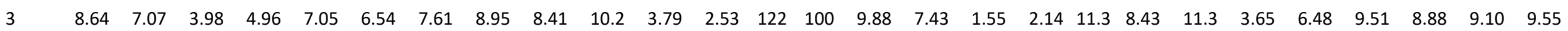

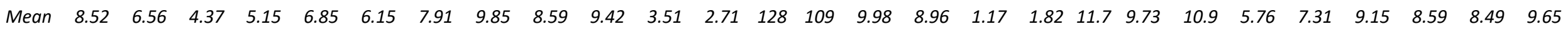




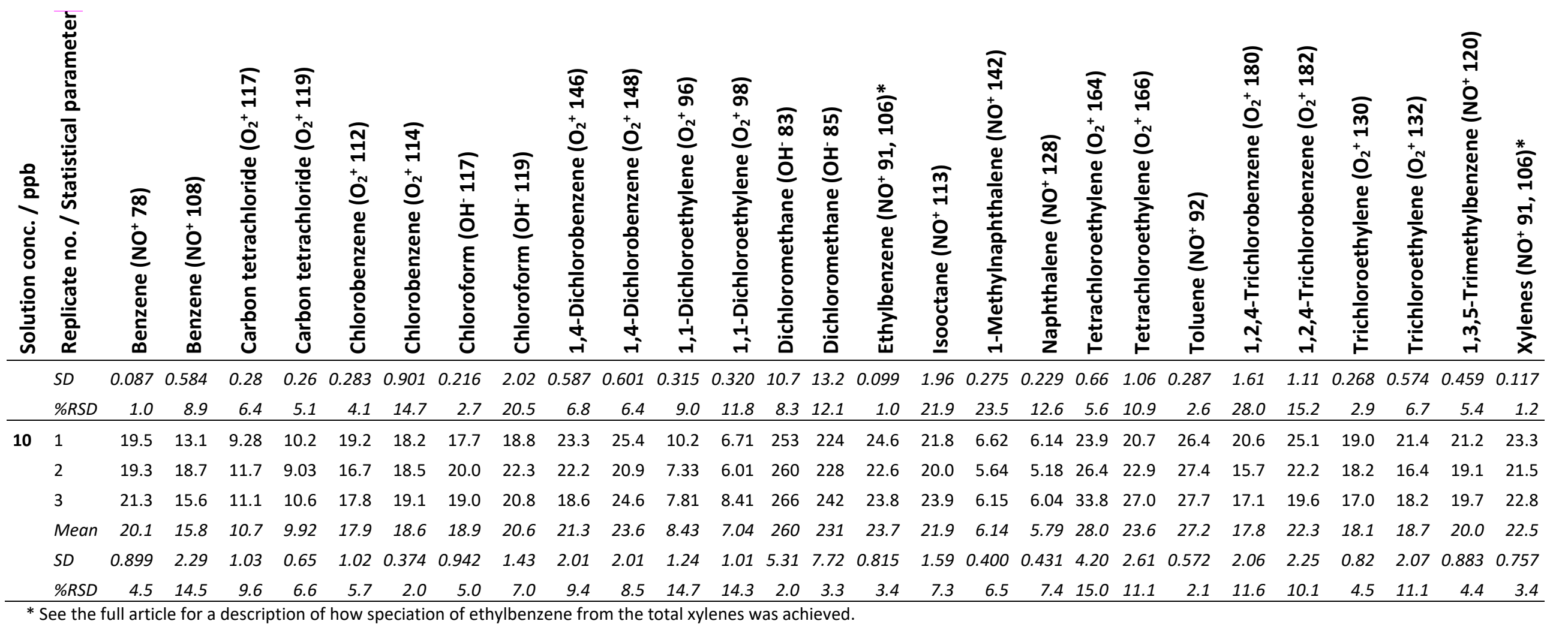


Table S7. System precision data for the method, with the mean and standard deviation shown in parts-per-million by volume measured in the headspace, and relative standard deviation (RSD) as a percentage.

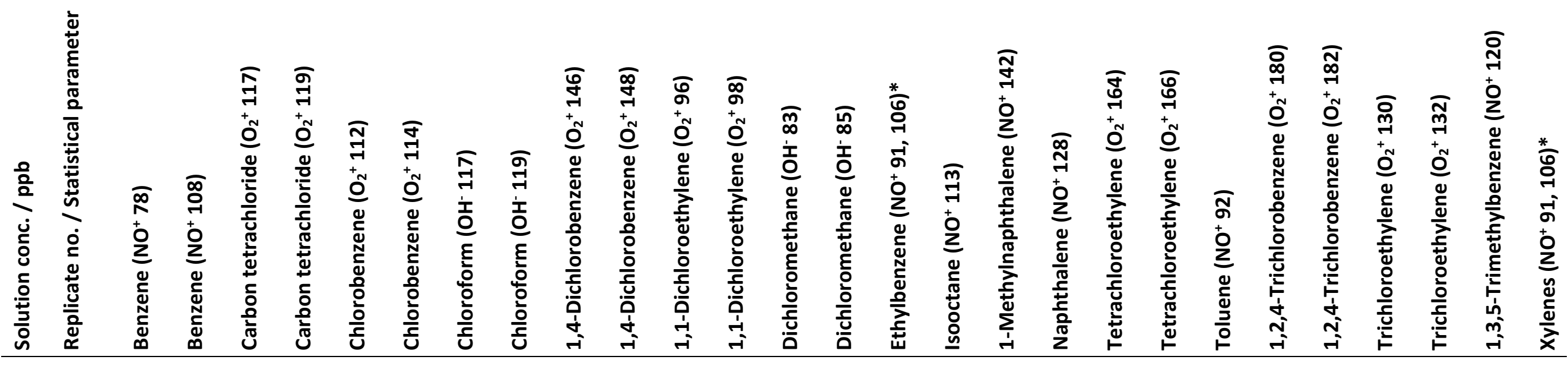

$\begin{array}{lllllllllllllllllllllllllllll}200 & 1 & 0.562 & 0.435 & 0.572 & 0.587 & 0.492 & 0.501 & 0.491 & 0.530 & 0.571 & 0.629 & 0.351 & 0.396 & 0.612 & 0.566 & 0.708 & 1.23 & 0.245 & 0.198 & 1.06 & 1.04 & 0.772 & 0.627 & 0.728 & 0.629 & 0.598 & 0.697 & 0.692\end{array}$

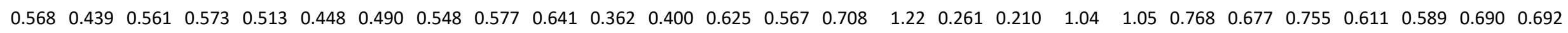
$\begin{array}{lllllllllllllllllllllllllll}0.530 & 0.404 & 0.497 & 0.531 & 0.497 & 0.453 & 0.488 & 0.501 & 0.566 & 0.633 & 0.338 & 0.348 & 0.563 & 0.528 & 0.670 & 1.13 & 0.256 & 0.212 & 0.961 & 0.943 & 0.735 & 0.645 & 0.707 & 0.594 & 0.528 & 0.652 & 0.650\end{array}$ $\begin{array}{lllllllllllllllllllllllllll}0.532 & 0.420 & 0.525 & 0.525 & 0.505 & 0.456 & 0.464 & 0.466 & 0.575 & 0.622 & 0.339 & 0.367 & 0.597 & 0.531 & 0.682 & 1.10 & 0.260 & 0.208 & 0.983 & 0.980 & 0.742 & 0.637 & 0.741 & 0.563 & 0.538 & 0.639 & 0.648\end{array}$ $\begin{array}{lllllllllllllllllllllllllll}0.589 & 0.442 & 0.583 & 0.595 & 0.529 & 0.500 & 0.504 & 0.518 & 0.576 & 0.637 & 0.379 & 0.416 & 0.616 & 0.546 & 0.724 & 1.21 & 0.251 & 0.202 & 1.11 & 1.06 & 0.812 & 0.647 & 0.768 & 0.614 & 0.631 & 0.707 & 0.706\end{array}$ $\begin{array}{llllllllllllllllllllllllllll}0.535 & 0.423 & 0.504 & 0.510 & 0.474 & 0.437 & 0.468 & 0.525 & 0.546 & 0.595 & 0.333 & 0.358 & 0.593 & 0.519 & 0.651 & 1.07 & 0.246 & 0.193 & 0.933 & 0.952 & 0.708 & 0.611 & 0.721 & 0.580 & 0.537 & 0.633 & 0.629\end{array}$ $\begin{array}{lllllllllllllllllllllllllllll}\text { Mean } & 0.553 & 0.428 & 0.541 & 0.554 & 0.501 & 0.466 & 0.484 & 0.514 & 0.568 & 0.626 & 0.350 & 0.381 & 0.601 & 0.543 & 0.691 & 1.16 & 0.253 & 0.204 & 1.01 & 1.00 & 0.757 & 0.641 & 0.737 & 0.598 & 0.570 & 0.669 & 0.669\end{array}$ $\begin{array}{lllllllllllllllllllllllllllll}S D & 0.022 & 0.013 & 0.033 & 0.033 & 0.017 & 0.025 & 0.014 & 0.026 & 0.011 & 0.015 & 0.016 & 0.025 & 0.020 & 0.019 & 0.025 & 0.063 & 0.006 & 0.007 & 0.061 & 0.047 & 0.033 & 0.020 & 0.021 & 0.022 & 0.038 & 0.029 & 0.028\end{array}$ \begin{tabular}{rrrrrrrrrrrrrrrrrrrrrrrrrrrrrrrr} 
& \%RSD & 4.0 & 3.1 & 6.2 & 5.9 & 3.4 & 5.4 & 2.9 & 5.0 & 1.9 & 2.4 & 4.6 & 6.5 & 3.4 & 3.4 & 3.6 & 5.4 & 2.5 & 3.3 & 6.0 & 4.7 & 4.3 & 3.2 & 2.8 & 3.7 & 6.7 & 4.4 & 4.2 \\
\hline 1000 & 1 & 2.77 & 2.11 & 3.05 & 3.14 & 2.58 & 2.36 & 2.80 & 3.05 & 2.74 & 2.95 & 1.89 & 2.01 & 3.18 & 2.98 & 3.73 & 6.44 & 1.18 & 0.958 & 5.45 & 5.46 & 3.98 & 3.16 & 3.77 & 3.02 & 2.97 & 3.72 & 3.53
\end{tabular}

$\begin{array}{lllllllllllllllllllllllllllllllllllll}2 & 2.72 & 2.13 & 2.97 & 3.08 & 2.49 & 2.41 & 2.70 & 2.94 & 2.76 & 2.96 & 1.81 & 1.94 & 3.16 & 2.90 & 3.64 & 5.79 & 1.18 & 0.954 & 5.46 & 5.51 & 3.86 & 3.11 & 3.58 & 3.05 & 3.05 & 3.59 & 3.56\end{array}$ $\begin{array}{lllllllllllllllllllllllllllll}2.77 & 2.12 & 3.03 & 3.06 & 2.61 & 2.47 & 2.73 & 2.87 & 2.77 & 3.07 & 1.83 & 2.02 & 3.14 & 2.85 & 3.68 & 6.25 & 1.23 & 0.986 & 5.39 & 5.37 & 3.93 & 3.22 & 3.82 & 3.06 & 2.99 & 3.59 & 3.57\end{array}$ $\begin{array}{lllllllllllllllllllllllllllll}2.78 & 2.14 & 3.08 & 3.16 & 2.55 & 2.43 & 2.84 & 2.96 & 2.67 & 3.06 & 1.85 & 2.04 & 3.16 & 2.99 & 3.78 & 6.44 & 1.23 & 0.969 & 5.51 & 5.60 & 3.96 & 3.19 & 3.72 & 3.15 & 3.05 & 3.73 & 3.65\end{array}$ $\begin{array}{lllllllllllllllllllllllllllllll}2.69 & 2.07 & 3.00 & 3.04 & 2.55 & 2.40 & 2.78 & 2.98 & 2.78 & 3.06 & 1.84 & 2.03 & 3.12 & 2.89 & 3.70 & 6.23 & 1.17 & 0.946 & 5.38 & 5.36 & 3.93 & 3.18 & 3.69 & 3.16 & 3.02 & 3.68 & 3.61\end{array}$ $\begin{array}{lllllllllllllllllllllllllllll}2.76 & 2.11 & 2.97 & 3.16 & 2.57 & 2.37 & 2.90 & 2.99 & 2.80 & 3.00 & 1.79 & 2.00 & 3.16 & 2.96 & 3.68 & 6.01 & 1.23 & 0.980 & 5.37 & 5.23 & 3.84 & 3.19 & 3.68 & 3.05 & 3.00 & 3.58 & 3.51\end{array}$ $\begin{array}{lllllllllllllllllllllllllllllll}\text { Mean } & 2.75 & 2.11 & 3.02 & 3.11 & 2.56 & 2.40 & 2.79 & 2.96 & 2.75 & 3.02 & 1.83 & 2.01 & 3.15 & 2.93 & 3.70 & 6.20 & 1.20 & 0.97 & 5.43 & 5.42 & 3.92 & 3.17 & 3.71 & 3.08 & 3.01 & 3.65 & 3.57\end{array}$ SD $\quad \begin{array}{llllllllllllllllllllllllllllll}0.032 & 0.022 & 0.041 & 0.049 & 0.037 & 0.037 & 0.066 & 0.054 & 0.041 & 0.049 & 0.031 & 0.032 & 0.019 & 0.051 & 0.045 & 0.232 & 0.027 & 0.014 & 0.051 & 0.119 & 0.051 & 0.034 & 0.075 & 0.053 & 0.030 & 0.064 & 0.046\end{array}$ \begin{tabular}{rrrrrrrrrrrrrrrrrrrrrrrrrrrrrr} 
& $\% R S D$ & 1.2 & 1.0 & 1.3 & 1.6 & 1.4 & 1.5 & 2.4 & 1.8 & 1.5 & 1.6 & 1.7 & 1.6 & 0.6 & 1.8 & 1.2 & 3.7 & 2.2 & 1.5 & 0.9 & 2.2 & 1.3 & 1.1 & 2.0 & 1.7 & 1.0 & 1.7 & 1.3 \\
\hline 1600 & 1 & 4.49 & 3.47 & 5.04 & 5.29 & 4.40 & 4.15 & 5.03 & 5.19 & 4.86 & 5.23 & 2.96 & 3.31 & 5.48 & 5.11 & 6.33 & 10.1 & 2.14 & 1.73 & 8.83 & 8.91 & 6.61 & 5.44 & 6.10 & 4.94 & 4.98 & 6.32 & 6.17
\end{tabular} $\begin{array}{lllllllllllllllllllllllllllllll}2 & 4.55 & 3.50 & 5.14 & 5.39 & 4.48 & 4.00 & 5.15 & 5.40 & 4.80 & 5.10 & 3.03 & 3.27 & 5.63 & 5.14 & 6.61 & 10.1 & 2.10 & 1.74 & 9.21 & 9.13 & 6.69 & 5.40 & 6.30 & 5.11 & 5.00 & 6.37 & 6.29\end{array}$ 


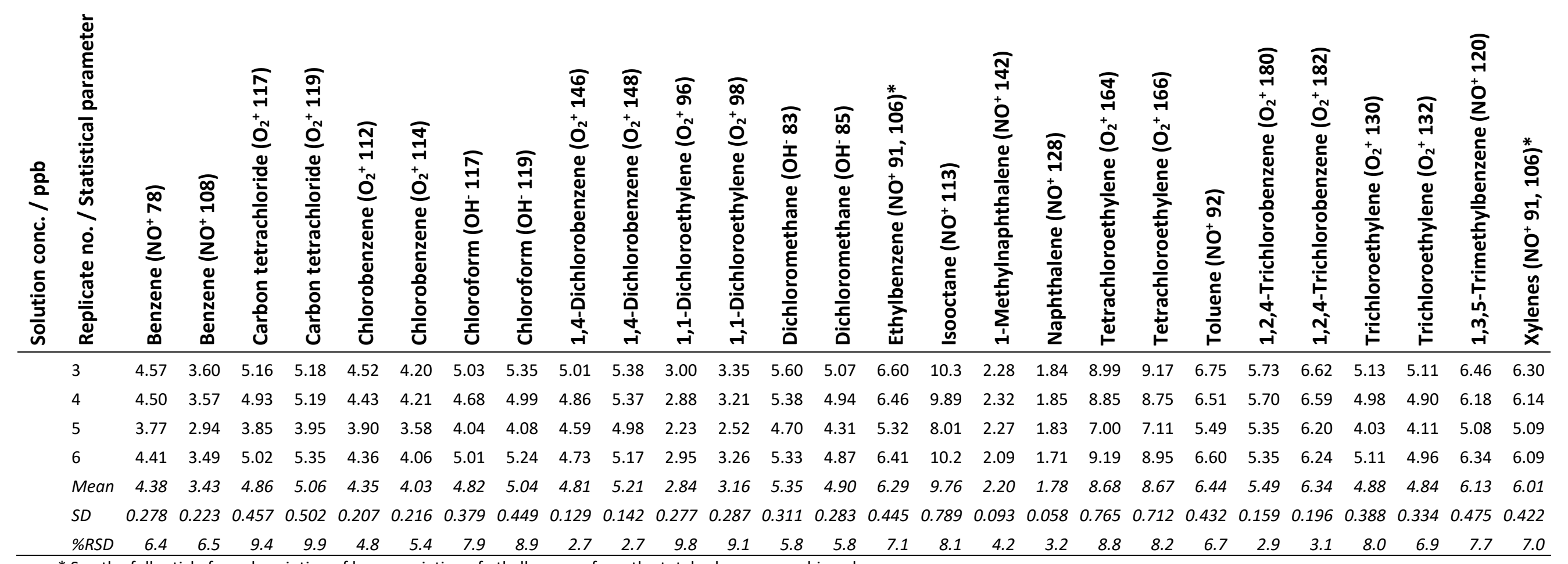

* See the full article for a description of how speciation of ethylbenzene from the total xylenes was achieved. 
Table 58. Analytical precision data for the method: (a) headspace concentrations in parts-per-million by volume measured in the headspace, and (b) corresponding concentration in solution (ppm), with mean, standard deviation (SD), and RSD (in \%) for the six replicates. The LOQs determined previously have been applied.

(a)

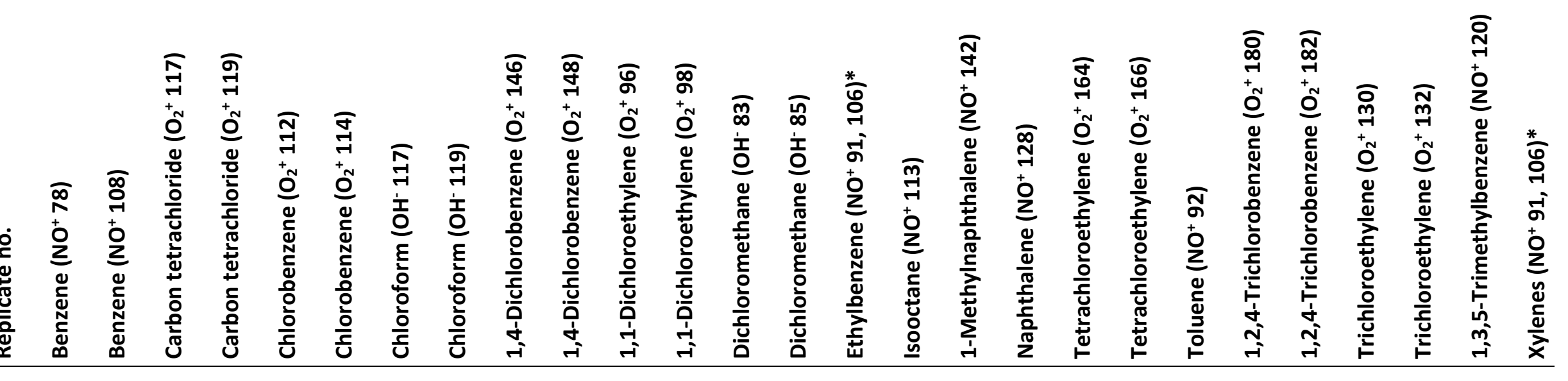

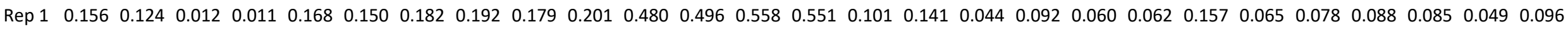

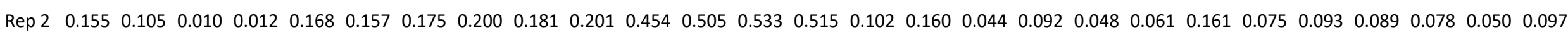
$\begin{array}{lllllllllllllllllllllllllllll}\text { Rep } 3 & 0.159 & 0.119 & 0.011 & 0.012 & 0.164 & 0.142 & 0.187 & 0.200 & 0.170 & 0.193 & 0.458 & 0.481 & 0.542 & 0.521 & 0.102 & 0.152 & 0.036 & 0.083 & 0.048 & 0.054 & 0.166 & 0.068 & 0.082 & 0.087 & 0.086 & 0.051 & 0.096\end{array}$ $\begin{array}{lllllllllllllllllllllllllllll}\text { Rep } 4 & 0.161 & 0.115 & 0.013 & 0.013 & 0.168 & 0.152 & 0.181 & 0.206 & 0.174 & 0.175 & 0.468 & 0.507 & 0.570 & 0.552 & 0.102 & 0.152 & 0.036 & 0.091 & 0.061 & 0.062 & 0.167 & 0.064 & 0.084 & 0.089 & 0.084 & 0.054 & 0.099\end{array}$

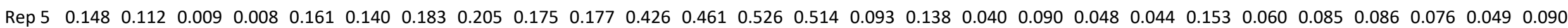
\begin{tabular}{llllllllllllllllllllllllllll} 
Rep 6 & 0.151 & 0.112 & 0.013 & 0.010 & 0.158 & 0.135 & 0.206 & 0.212 & 0.167 & 0.174 & 0.431 & 0.459 & 0.588 & 0.564 & 0.099 & 0.158 & 0.036 & 0.083 & 0.053 & 0.052 & 0.154 & 0.060 & 0.067 & 0.080 & 0.086 & 0.049 & 0.096 \\
\hline
\end{tabular} 
(b)

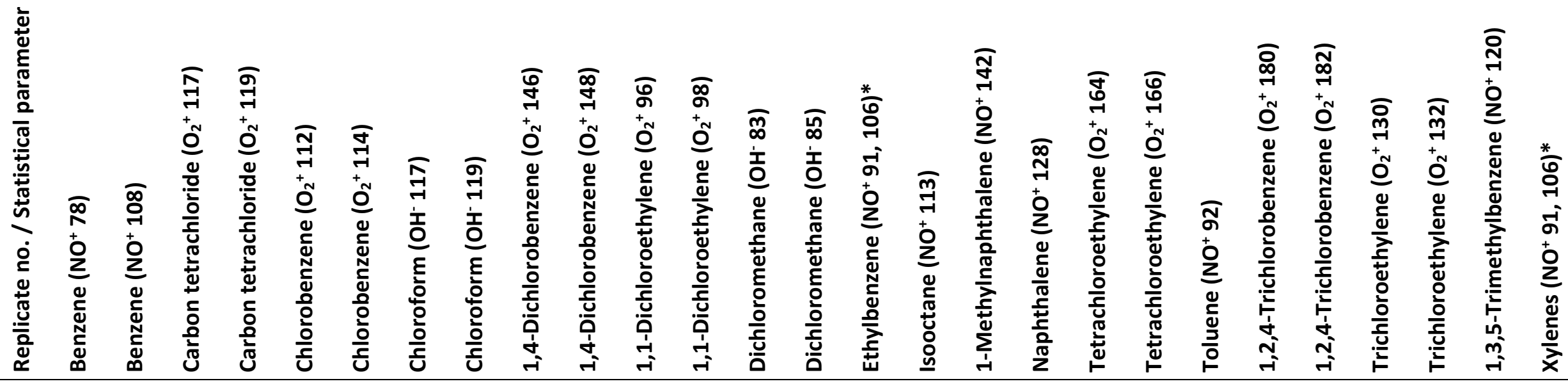

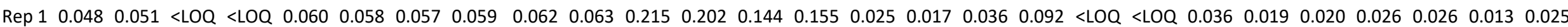
$\begin{array}{lllllllllllllllllllllllllllll}\text { Rep } 2 & 0.048 & 0.044 & <\text { LOQ }<\text { LOQ } & 0.060 & 0.060 & 0.055 & 0.061 & 0.063 & 0.063 & 0.203 & 0.206 & 0.137 & 0.145 & 0.025 & 0.019 & 0.036 & 0.092 & <\text { LOQ } & <\text { LOQ } & 0.037 & 0.022 & 0.024 & 0.026 & 0.024 & 0.013 & 0.025\end{array}$ $\begin{array}{llllllllllllllllllllllllllllllllll}\text { Rep } 3 & 0.049 & 0.049 & <\text { LOQ }<\text { LOQ } & 0.059 & 0.055 & 0.059 & 0.061 & 0.059 & 0.061 & 0.205 & 0.196 & 0.140 & 0.146 & 0.025 & 0.018 & 0.029 & 0.082 & <\text { LOQ } & <\text { LOQ } & 0.038 & 0.020 & 0.021 & 0.025 & 0.026 & 0.013 & 0.025\end{array}$

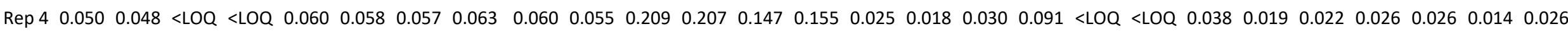

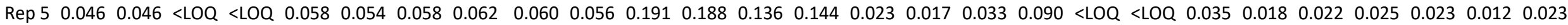
$\begin{array}{llllllllllllllllllllllllllllll}\text { Rep } 6 & 0.046 & 0.046 & <\text { LOQ }<\text { LOQ } & 0.057 & 0.052 & 0.065 & 0.065 & 0.058 & 0.055 & 0.193 & 0.187 & 0.151 & 0.159 & 0.024 & 0.019 & 0.029 & 0.082 & <\text { LOQ } & <\text { LOQ } & 0.035 & 0.018 & 0.017 & 0.023 & 0.026 & 0.012 & 0.025\end{array}$

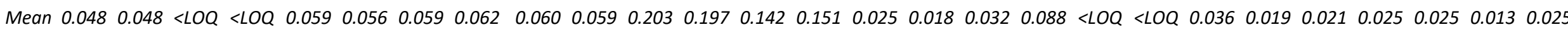
$\begin{array}{llllllllllllllllllllllllllllllll}S D & 0.001 & 0.003 & N / A & N / A & 0.001 & 0.003 & 0.003 & 0.002 & 0.002 & 0.004 & 0.009 & 0.008 & 0.006 & 0.006 & 0.001 & 0.001 & 0.003 & 0.004 & N / A & N / A & 0.001 & 0.002 & 0.002 & 0.001 & 0.001 & 0.001 & 0.001\end{array}$

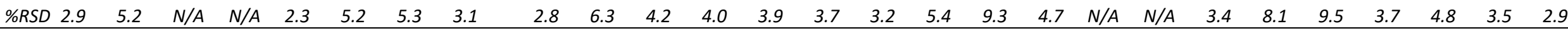
* See the full article for a description of how speciation of ethylbenzene from the total xylenes was achieved. 
Table S9. Accuracy data (in ppm in solution) for each of the triplicate measurements at 500, 1000, and 1500 ppb concentration in water, together with mean, standard deviation (SD), and RSD (in \%).

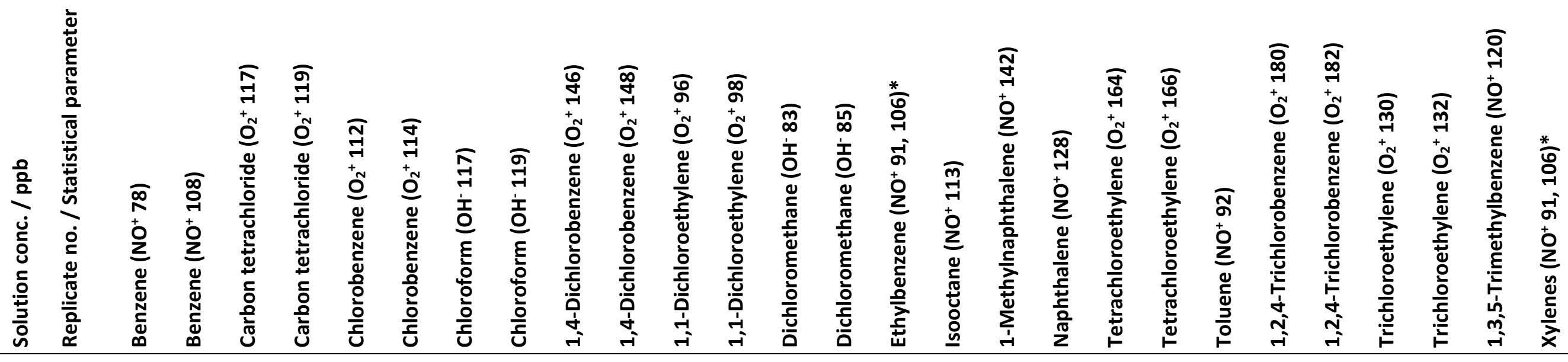

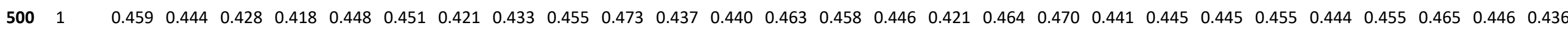

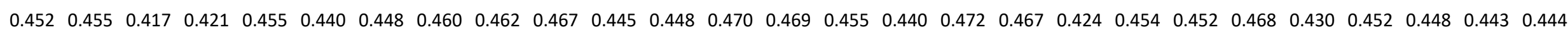

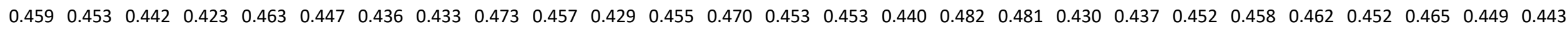

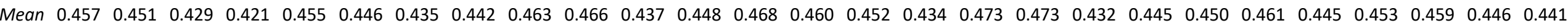

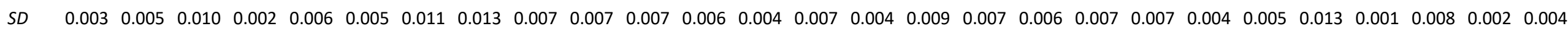

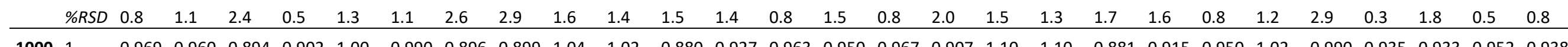

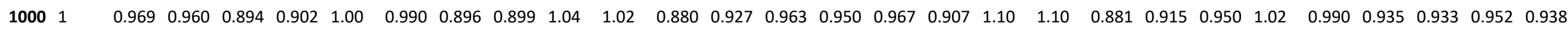

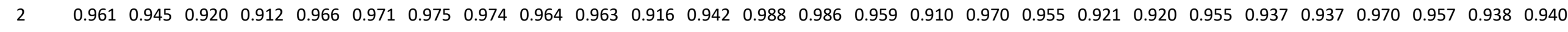

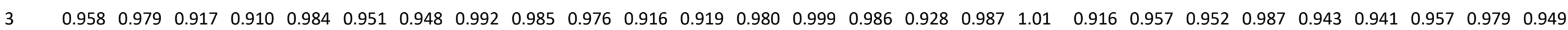

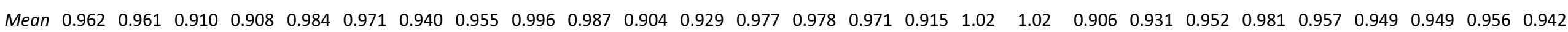

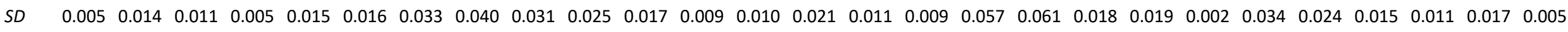

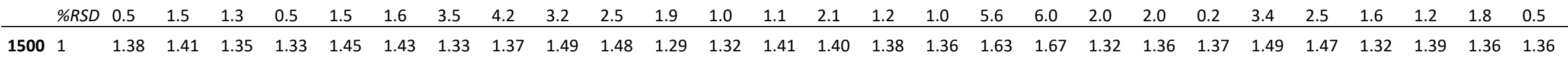

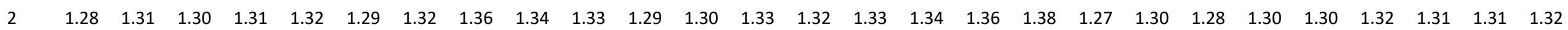

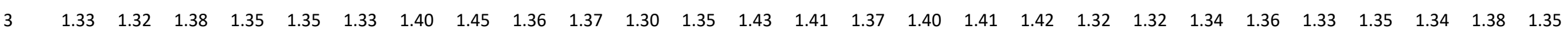

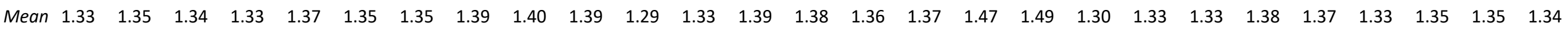

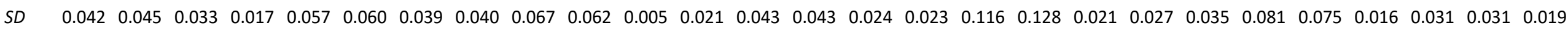

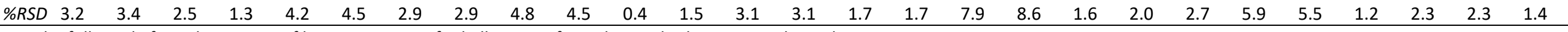
* See the full article for a description of how speciation of ethylbenzene from the total xylenes was achieved. 
Table S10. Recovery data (\%) for each of the triplicate measurements at 500, 1000, and 1500 ppb concentration in water, together with mean, standard deviation (SD), and RSD (in \%).

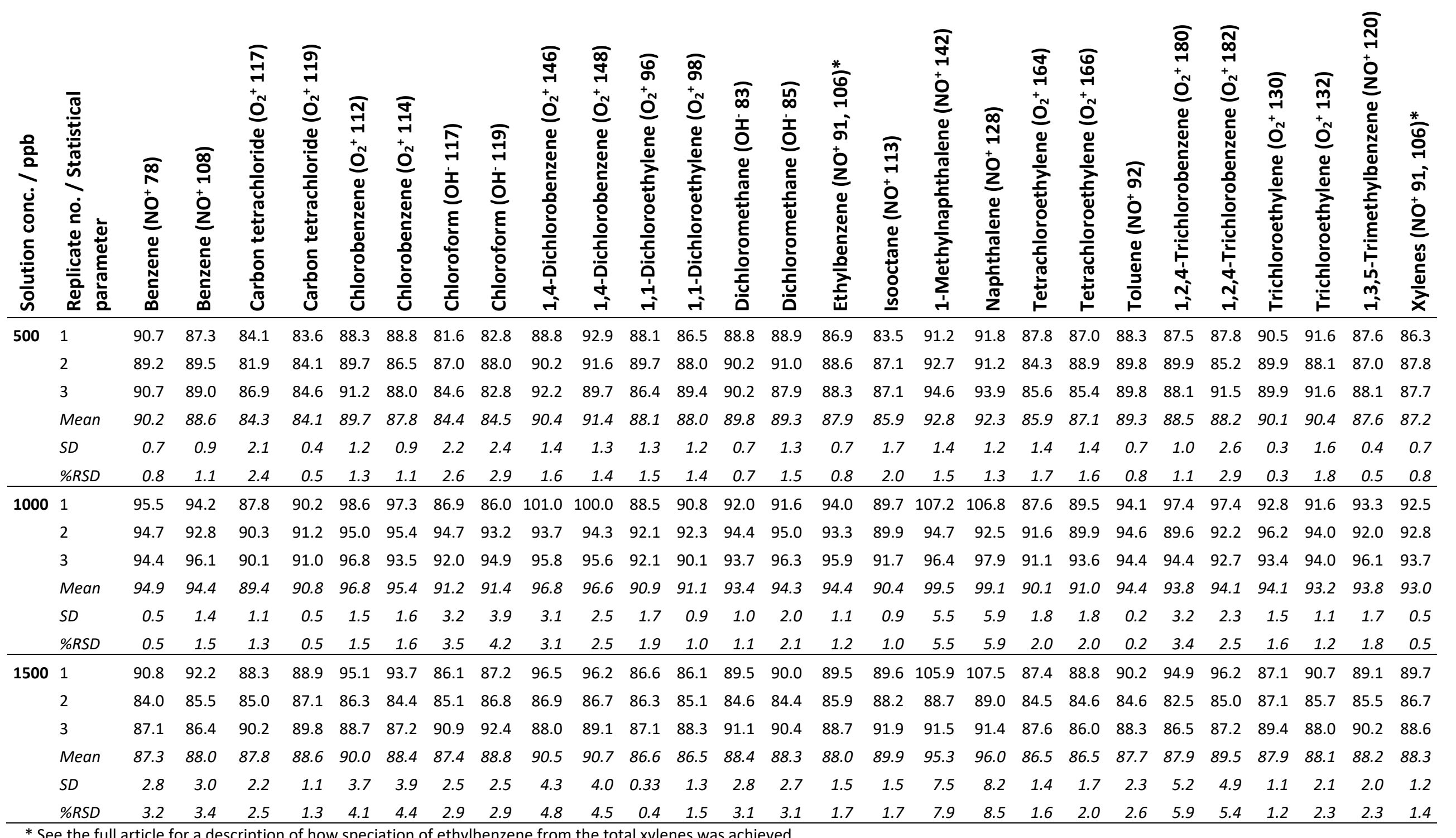


Table S11. Accuracy data (in ppm in solution) obtained in the intermediate precision study (different analyst). Triplicate measurements were made at 500, 1000 , and 1500 ppb concentration in water. Means, standard deviations (SD), and RSDs (in \%) are also shown.

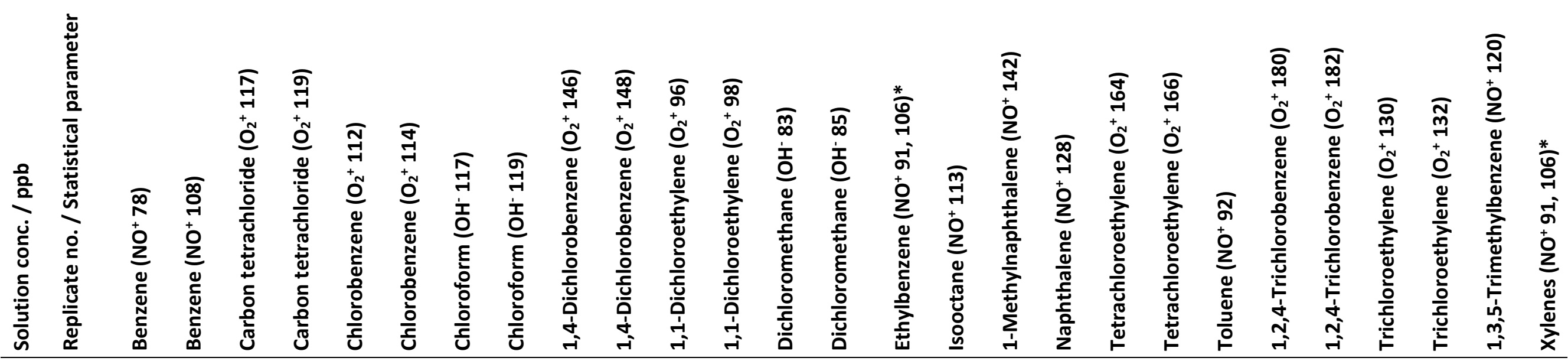

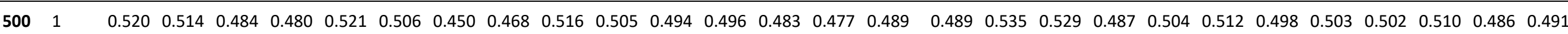

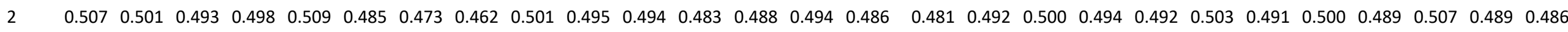

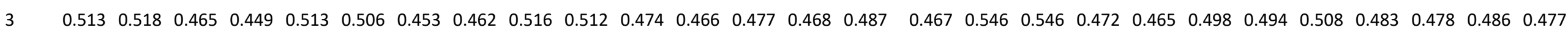
$\begin{array}{lllllllllllllllllllllllllllll}\text { Mean } & 0.513 & 0.511 & 0.480 & 0.476 & 0.514 & 0.499 & 0.459 & 0.464 & 0.511 & 0.504 & 0.487 & 0.481 & 0.483 & 0.480 & 0.487 & 0.479 & 0.524 & 0.525 & 0.485 & 0.487 & 0.504 & 0.494 & 0.504 & 0.491 & 0.498 & 0.487 & 0.485\end{array}$ SD $\quad \begin{array}{llllllllllllllllllllllllllllllll}0.005 & 0.008 & 0.012 & 0.020 & 0.005 & 0.010 & 0.010 & 0.003 & 0.007 & 0.007 & 0.010 & 0.012 & 0.005 & 0.011 & 0.0014 & 0.009 & 0.023 & 0.019 & 0.009 & 0.017 & 0.006 & 0.003 & 0.004 & 0.008 & 0.014 & 0.001 & 0.006\end{array}$ \begin{tabular}{llllllllllllllllllllllllllll} 
\%RSD & 1.1 & 1.5 & 2.5 & 4.2 & 1.0 & 2.0 & 2.2 & 0.6 & 1.4 & 1.4 & 2.0 & 2.6 & 0.9 & 2.3 & 0.3 & 1.8 & 4.4 & 3.6 & 1.9 & 3.4 & 1.2 & 0.5 & 0.7 & 1.6 & 2.9 & 0.3 & 1.2 \\
\hline
\end{tabular} $\begin{array}{llllllllllllllllllllllllllllll}1000 & 1 & 0.942 & 0.941 & 0.863 & 0.860 & 0.996 & 1.01 & 0.881 & 0.877 & 1.04 & 1.04 & 0.885 & 0.879 & 0.919 & 0.916 & 0.882 & 0.875 & 1.17 & 1.17 & 0.860 & 0.876 & 0.927 & 1.04 & 1.06 & 0.912 & 0.900 & 0.901 & 0.881 \\ & 2 & 0.985 & 0.981 & 1.01 & 0.996 & 1.008 & 0.994 & 0.983 & 1.014 & 0.997 & 0.980 & 0.971 & 0.930 & 0.987 & 0.969 & 0.993 & 0.959 & 1.02 & 1.02 & 0.971 & 0.986 & 0.982 & 0.960 & 0.981 & 0.955 & 0.986 & 0.991 & 0.993\end{array}$ $\begin{array}{lllllllllllllllllllllllllll}1.042 & 1.02 & 0.943 & 0.954 & 1.04 & 1.023 & 0.934 & 0.934 & 1.05 & 1.07 & 0.932 & 0.913 & 0.946 & 0.937 & 0.986 & 0.949 & 1.17 & 1.18 & 0.946 & 0.943 & 0.979 & 1.06 & 1.09 & 0.965 & 0.970 & 0.977 & 0.987\end{array}$

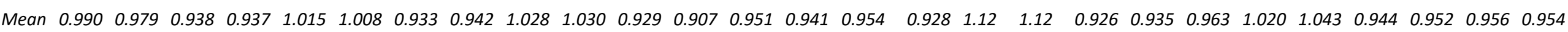
SD $\quad \begin{array}{lllllllllllllllllllllllllllll}0.041 & 0.031 & 0.060 & 0.057 & 0.018 & 0.012 & 0.042 & 0.056 & 0.023 & 0.038 & 0.035 & 0.022 & 0.028 & 0.022 & 0.051 & 0.037 & 0.074 & 0.075 & 0.048 & 0.045 & 0.025 & 0.043 & 0.045 & 0.023 & 0.038 & 0.040 & 0.051\end{array}$ \begin{tabular}{lllllllllllllllllllllllllllll} 
& $\% R S D$ & 4.1 & 3.2 & 6.3 & 6.1 & 1.8 & 1.2 & 4.5 & 5.9 & 2.2 & 3.7 & 3.8 & 2.4 & 2.9 & 2.3 & 5.3 & 4.0 & 6.6 & 6.6 & 5.1 & 4.8 & 2.6 & 4.2 & 4.3 & 2.4 & 3.9 & 4.1 & 5.4 \\
\hline 1500 & 1 & 1.54 & 1.51 & 1.56 & 1.54 & 1.60 & 1.59 & 1.51 & 1.53 & 1.59 & 1.56 & 1.47 & 1.46 & 1.50 & 1.46 & 1.58 & 1.48 & 1.68 & 1.69 & 1.52 & 1.51 & 1.57 & 1.55 & 1.63 & 1.47 & 1.52 & 1.57 & 1.54
\end{tabular}

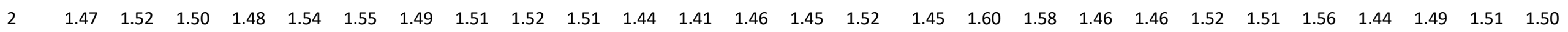

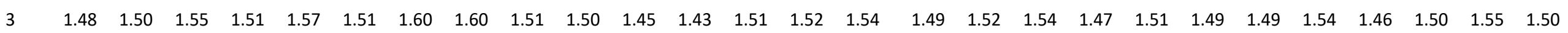
$\begin{array}{llllllllllllllllllllllllllll}\text { Mean } & 1.50 & 1.51 & 1.54 & 1.51 & 1.57 & 1.55 & 1.53 & 1.55 & 1.54 & 1.53 & 1.45 & 1.44 & 1.49 & 1.48 & 1.545 & 1.473 & 1.60 & 1.60 & 1.48 & 1.49 & 1.53 & 1.52 & 1.58 & 1.46 & 1.51 & 1.55 & 1.51\end{array}$

$\begin{array}{llllllllllllllllllllllllllllll}S D & 0.030 & 0.011 & 0.026 & 0.026 & 0.024 & 0.033 & 0.048 & 0.042 & 0.039 & 0.025 & 0.013 & 0.022 & 0.019 & 0.030 & 0.027 & 0.017 & 0.068 & 0.066 & 0.026 & 0.022 & 0.035 & 0.023 & 0.038 & 0.012 & 0.013 & 0.028 & 0.020\end{array}$ $\begin{array}{lllllllllllllllllllllllllllll}\text { \%RSD } & 2.0 & 0.7 & 1.7 & 1.7 & 1.6 & 2.1 & 3.1 & 2.7 & 2.5 & 1.7 & 0.9 & 1.5 & 1.3 & 2.0 & 1.7 & 1.1 & 4.2 & 4.1 & 1.7 & 1.4 & 2.3 & 1.5 & 2.4 & 0.9 & 0.9 & 1.8 & 1.3\end{array}$

* See the full article for a description of how speciation of ethylbenzene from the total xylenes was achieved. 
Table S12. Recovery data (\%) obtained in the intermediate precision study (different analyst). Triplicate measurements were made at 500, 1000, and 1500 ppb concentration in water. Means, standard deviations (SD), and RSDs (in \%) are also shown.

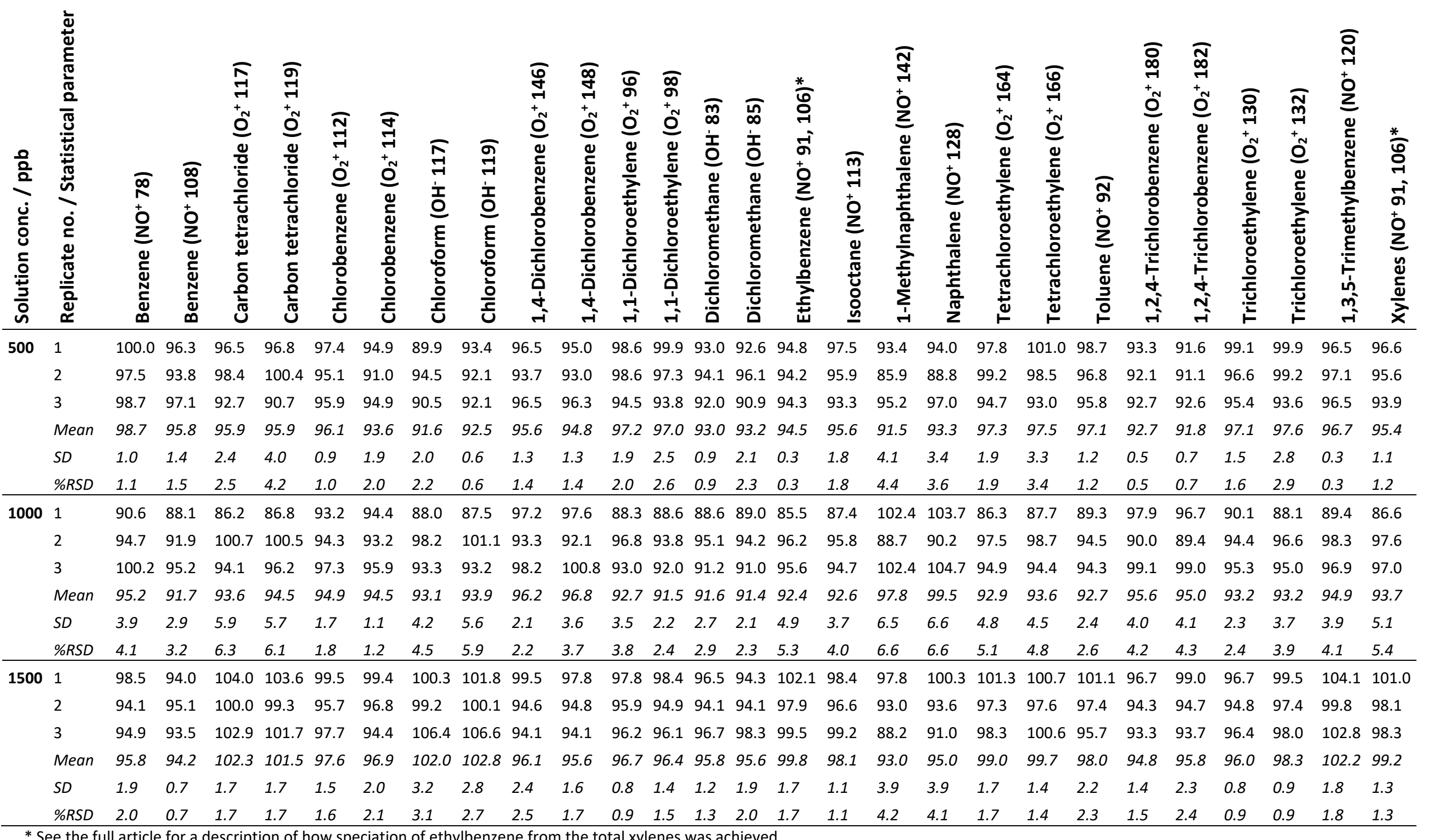


Table S13. Robustness data as assessed using linearity $\left(\mathrm{R}^{2}\right)$, repeatability (\%RSD), and recovery (percentage relative to calibration at $150{ }^{\circ} \mathrm{C}$ and $25 \mathrm{sccm}$; $\%$ Rec.) measures for two changes to sample flow rate and inlet temperature reduction.

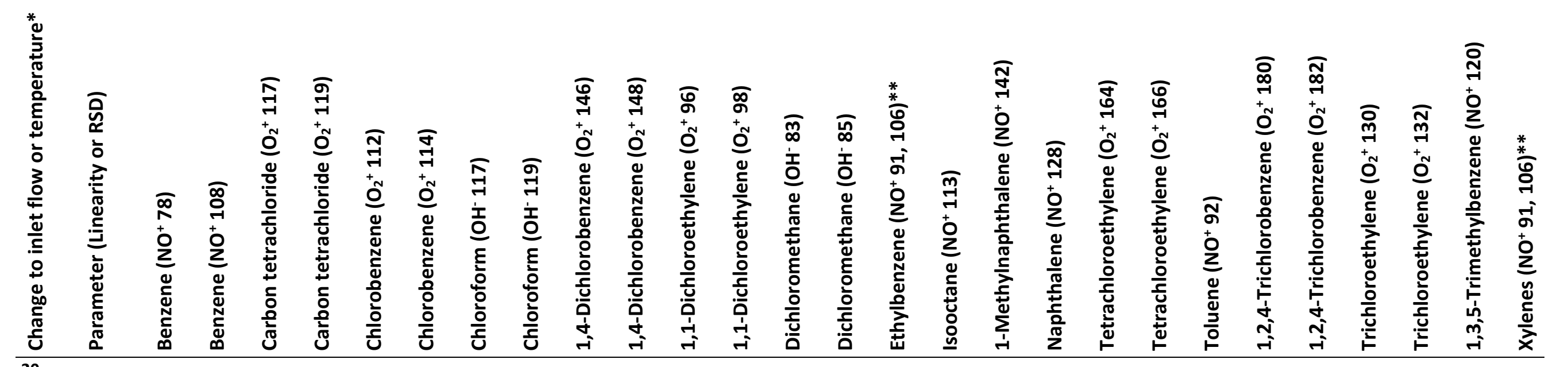

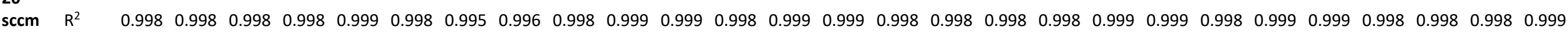

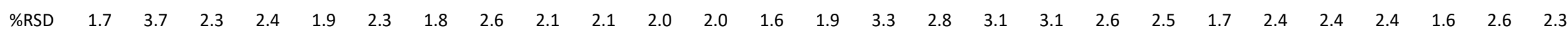

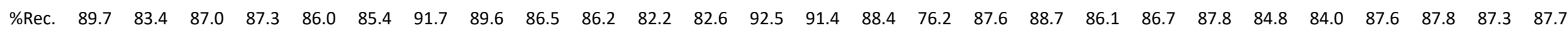
30

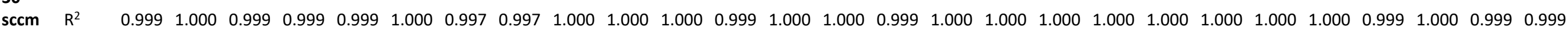

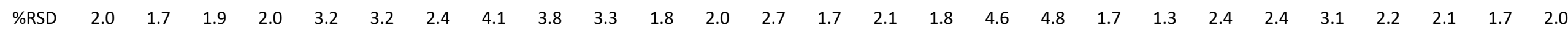

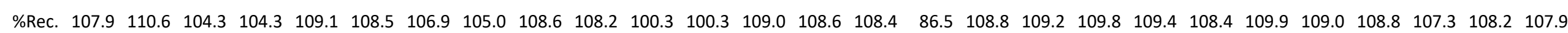

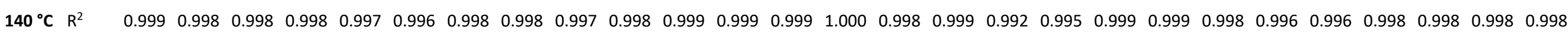

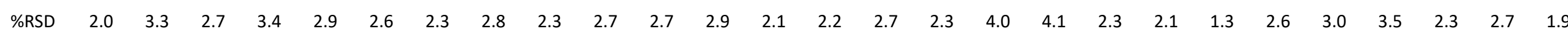

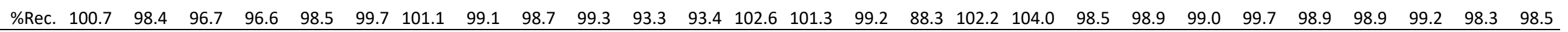

* Standard inlet flow is ca. $25 \mathrm{sccm}$ and inlet temperature is $150{ }^{\circ} \mathrm{C}$.

** See the full article for a description of how speciation of ethylbenzene from the total xylenes was achieved. 